\title{
Anti-glioblastoma Effects of Structural Variants of Benzoylphenoxyacetamide (BPA): II. Synthesis Strategies for Phenolic Variants of BPAs With Potential for Blood Brain Barrier Penetration.
}

\section{Joanna Stalinska}

Louisiana State University Health Sciences Center, Stanley S. Scott Cancer Center, Department of Interdisciplinary Medicine, New Orleans, LA 70112

\section{Cecilia Vittori}

Louisiana State University Health Sciences Center, Stanley S. Scott Cancer Center, Department of Interdisciplinary Medicine, New Orleans, LA 70112

Charles H. Ingraham IV

Louisiana State University Health Sciences Center, Stanley S. Scott Cancer Center, Department of Interdisciplinary Medicine, New Orleans, LA 70112

\section{Sean C. Carson}

University of New Orleans

\section{Karlie Bonstaff}

Louisiana State University Health Sciences Center, Stanley S. Scott Cancer Center, Department of Interdisciplinary Medicine, New Orleans, LA 70112

\section{Adam Lassak}

Louisiana State University Health Sciences Center, Stanley S. Scott Cancer Center, Department of Interdisciplinary Medicine, New Orleans, LA 70112

\section{Celeste Faia}

Louisiana State University Health Sciences Center, Stanley S. Scott Cancer Center, Department of Interdisciplinary Medicine, New Orleans, LA 70112

\section{Susan B. Colley}

Louisiana State University Health Sciences Center, Stanley S. Scott Cancer Center, Department of Interdisciplinary Medicine, New Orleans, LA 70112

\section{Francesca Peruzzi}

Louisiana State University Health Sciences Center, Stanley S. Scott Cancer Center, Department of Interdisciplinary Medicine, New Orleans, LA 70112

\section{Krzysztof Reiss ( $\sim$ kreiss@lsuhsc.edu )}

Louisiana State University Health Sciences Center, Stanley S. Scott Cancer Center, Department of Interdisciplinary Medicine, New Orleans, LA 70112

\section{Branko S. Jursic}




\section{Research Article}

Keywords: temozolomide (TMZ), MPO-CNS, logS, blood-brain partitioning, surgical resection

Posted Date: September 21st, 2021

DOl: https://doi.org/10.21203/rs.3.rs-909472/v1

License: (c) (i) This work is licensed under a Creative Commons Attribution 4.0 International License. Read Full License 
Anti-glioblastoma effects of structural variants of benzoylphenoxyacetamide (BPA): II. Synthesis strategies for phenolic variants of BPAs with potential for Blood Brain Barrier penetration.

Joanna Stalinska ${ }^{c, d}$, Cecilia Vittoric,e, Charles H. Ingraham IV ${ }^{\mathrm{a}}$, Sean C. Carsona ${ }^{\mathrm{a}}$, Karlie Bonstaffc, Adam Lassakc, Celeste Faia ${ }^{c}$, Susan B. Colleyc ${ }^{c}$, Francesca Peruzzic, Krzysztof Reiss ${ }^{c^{*}}$, and Branko S. Jursic ${ }^{a, b^{*}}$

${ }^{a}$ Department of Chemistry, University of New Orleans, New Orleans, LA 70148, United States; 'b ${ }^{2}$ tepharm Ilc., PO Box 24220, New Orleans, LA 70184, United States; 'Neurological Cancer Research, Stanley S. Scott Cancer Center, Department of Medicine, LSU Health Sciences Center, New Orleans, LA 70112; dDepartment of Cell Biology, Faculty of Biochemistry, Biophysics and Biotechnology, Jagiellonian University,

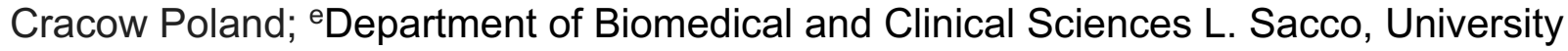
of Milan, Milan, Italy.

*corresponding authors 


\section{ABSTRACT}

Glioblastomas are the most aggressive brain tumors for which therapeutic options are limited. Current therapies against glioblastoma include surgical resection, followed by radiotherapy plus concomitant and maintenance with temozolomide (TMZ), however, these standard therapies are often ineffective, and average survival time for glioblastoma patients is between 12 and 18 months. We have previously reported a strong antiglioblastoma activity of several metabolic compounds, which were synthetized based the chemical structure of a common lipid-lowering drug, fenofibrate, and share a general molecular skeleton of benzoylphenoxyacetamide (BPA). Extensive computational analyses of phenol and naphthol moieties added to the BPA skeleton were performed in this study with the objective of selecting new BPA variants for subsequent compound preparation and anti-glioblastoma testing. Initially, 81 structural variations were considered and their physical properties such as solubility (logS), blood-brain partitioning ( $\log B B)$, and probability of entering the CNS calculated by the Central Nervous System Multiparameter Optimization (MPO-CNS) algorithm were evaluated. From this initial list, 18 compounds were further evaluated for anti-glioblastoma activity in vitro. Nine compounds demonstrated desirable glioblastoma cell toxicity in cell culture, and two of them, HR51, and HR59 demonstrated significantly improved capability of crossing the model blood-brain-barrier (BBB) composed of endothelial cells, astrocytes and pericytes. 


\section{INTRODUCTION}

Glioblastomas are the most aggressive brain tumors for which therapeutic options are very limited ${ }^{1,2}$. Current standard of care therapies include maximal surgical resection, followed by radiotherapy plus concomitant and maintenance with temozolomide (TMZ), however, these standard therapies are often ineffective, contributing to the dismal glioblastoma patient survival time of $12-18$ months ${ }^{3}$. Multiple genetic and epigenetic abnormalities have been found in glioblastomas, among which p53, EGFR, PTEN, and IDH mutations are the most common ${ }^{4-6}$. In spite of these validated therapeutic targets, molecular, gene-therapy, and immunotherapy approaches are still ineffective ${ }^{7,8}$. Therefore, new and more effective therapies for glioblastoma patients are desperately needed.

There are several reasons why it is difficult to treat glioblastoma. First, glioblastomas are characterized by many dysregulated pathways that cannot be blocked simultaneously with a single therapy ${ }^{9}$; Second, glioblastomas are highly infiltrating and create heterogenous tumors that are very difficult to be removed by surgery without compromising the function of the surrounding brain areas ${ }^{10}$; Third, early diagnosis of glioblastoma is rare, therefore, large highly infiltrating and vascularized tumors are often already present at the time of diagnosis ${ }^{11}$. Fourth, the optimization of clinical protocols for glioblastoma treatment requires the use of a reliable preclinical model/s. Unfortunately, commonly used rodent syngeneic and xenograft models have one major problem - the experimental tumors are typically $\sim 10^{3}-10^{4}$ smaller than human tumors, and therefore, drug delivery, drug retention, and effective tissue penetration by the drug, cannot be tested in a reliable manner in small animal models ${ }^{12}$; Fifth, the blood brain barrier (BBB) 
prevents the majority of anticancer drugs from reaching the tumor site, and current methods that enhance the BBB penetration are not effective for glioblastoma patients ${ }^{13}$.

One of the drugs that readily crosses the BBB is temozolomide (TMZ). Upon oral administration, TMZ maximum plasma concentration can be reached in about one hour, and the elimination half-life is approximately 2.1 hours. Importantly, penetration efficiency of TMZ into the CNS is experimentally estimated to be about $20 \%$ of the plasma levels ${ }^{14}$. Applying this estimate to calculate the logBB (Brain-Blood Distribution) for TMZ, this equation produces a value of -0.7 , which indicates sufficient capability of the compound to cross the BBB ${ }^{15}$. In spite of these positive features, TMZ-treated glioblastoma patients develop TMZ-resistance and recurrent tumors are practically incurable ${ }^{16}$. There are also several studies of the use of TMZ in combination with other drugs, which show beneficial therapeutic effects ${ }^{17} 18$. One interesting example is a combination of TMZ with lipid lowering drugs, including statins ${ }^{19}$. In addition, another class of lipid-lowering compounds, fibrates, have also attracted attention as a possible anticancer drugs ${ }^{20-23}$. We have previously reported that $50 \mu \mathrm{M}$ fenofibrate (FF) has a strong anti-glioblastoma activity in cell culture, and in glioblastoma mouse models following intratumoral injection ${ }^{24}$ (Scheme 1). However, FF does not cross the BBB, and is quickly processed by the blood and tissue esterases to form fenofibric acid (FFA), which is no longer effective in triggering tumor cell death ${ }^{24,25}$.

We have previously made several chemical modifications to the FF molecular skeleton, to address the FF low stability in human blood, low water solubility, and inability of penetrating the BBB. Indeed, one of the initial compounds, PP1, demonstrated improved water solubility and stability in human blood. In addition, PP1 was capable of 
triggering extensive glioblastoma cell death in vitro at concentrations over 4-fold lower than FF ${ }^{26}$ (Scheme 1). To further improve anti-glioblastoma efficacy, we created other FF derivatives, which share the benzoyl-phenoxy-acetamide (BPA) molecular skeleton, and decided to test the addition of phenol and naphthol residues to the BPA structure due to the potential anti-cancer effects of these moieties ${ }^{27-30}$. As a result, 18 new compounds were generated and were analyzed during this study.

\begin{tabular}{|l|c|c|c|}
\hline & LF & Moderate & Moderate \\
\hline Water Solubility & LoW & High & High \\
\hline Resistance to blood esterases & $\mathrm{IC} 50^{*}=31.83 \mu \mathrm{M}$ & No & $\mathrm{IC} 50^{*}=7.7 \mu \mathrm{M}$ \\
\hline Antitumoral effect &
\end{tabular}

Scheme 1. Comparison between FF, FFA, and PP1 structural and anti-cancer properties. The information regarding the compounds water solubility, stability in human blood, and in vitro cytotoxicity were previously reported ${ }^{31}$. "determined using LN229 monolayer cultures and MTT assay.

\section{RESULTS and DISCUSSON}

Overall Chemical Design: In our previous studies we have explored the importance of a basic BPA skeleton ${ }^{31}$, and concluded that BPA could serve as a "pharmacophore", necessary to retain anti-glioblastoma activity ${ }^{32,33}$. The amide part of the BPA skeleton can be specifically modelled to obtain a more desirable anti-tumor activity. This includes, among other properties, chemical and physical parameters (described below) that contribute to the increased BBB penetration, and possibly drug retention within the tumor tissue. In this regard, we have selected phenol and naphthol residues due to mounting evidence supporting the role of different derivatives of these compounds in health benefits ${ }^{34}$, including anti-cancer activities ${ }^{28,29}$. In this paper, three variants of BPA are discussed: a substituted phenol (Phenolic-BPA)], and two naphtholic BPAs (1-Naphtholic-BPA and 
2-Naphtholic-BPA) (Scheme 2) that serve as prototype molecules for further modifications.
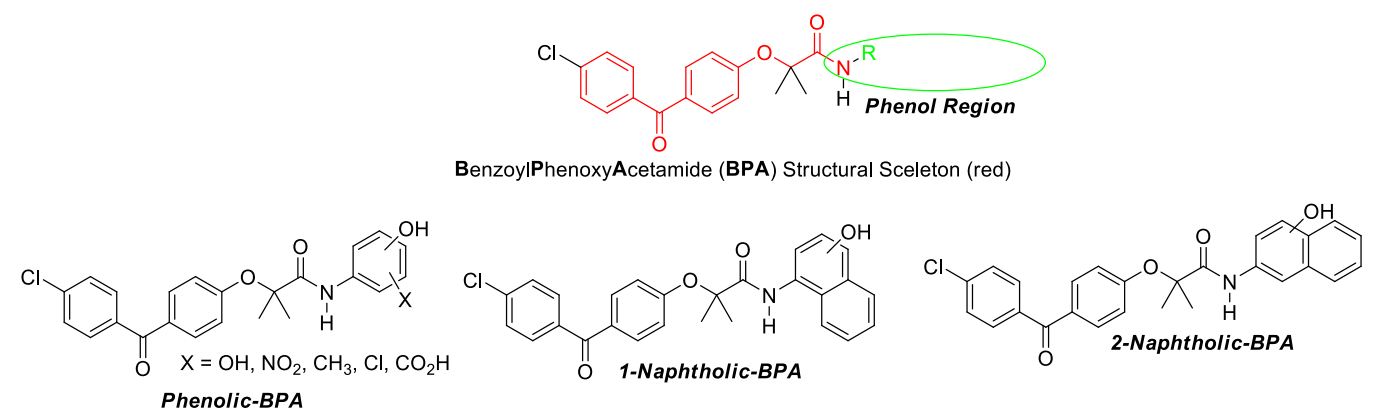

Scheme 2. Phenol region of BPA skeleton selected for modification (circle) in search of the optimal antiglioblastoma drug.

The starting point for the preparation of all phenolic BPAs is fenofibric acid (FFA), and the corresponding aminophenol or aminonaphthol residues (Scheme 3) are added through amide (peptide) coupling reactions ${ }^{35,36}$. As previously reported ${ }^{31}$, due to the steric hindrance of the carboxylic group of FFA, which includes two methyl groups in the alpha position of carboxylic acid, combined with the lower amine nucleophilicity of anilines in DCC- or EDC-coupling, these reactions do not produce acceptable isolated yields. This occurs even with more reactive aminophenols and EDC or DCC, which are stronger nucleophiles compared to nonactivated anilines, which is expected to produce corresponding BPA compounds in acceptable yields ${ }^{37}$. However, we were able to detect only traces of the desirable products with these methods, and instead, decided to convert FFA into the more reactive fenofibrate chloride (FFC), followed by coupling with aminophenols or aminonaphthols (Scheme 3). We have explored several variations of this procedure and finally selected one that is very simple and can be applied to multigram and even multikilogram production scales. In particular, the FFC was prepared fresh and immediately used, for the next step of aminophenol addition (Scheme 3). The most 
common method of preparation of an acid chloride is by using thionyl chloride. This requires heating of thionyl chloride with the corresponding acid (in this case FFA) with appropriate traps for hydrochloric acid and sulfur dioxide, which are undesirable biproducts of the reaction ${ }^{38}$.

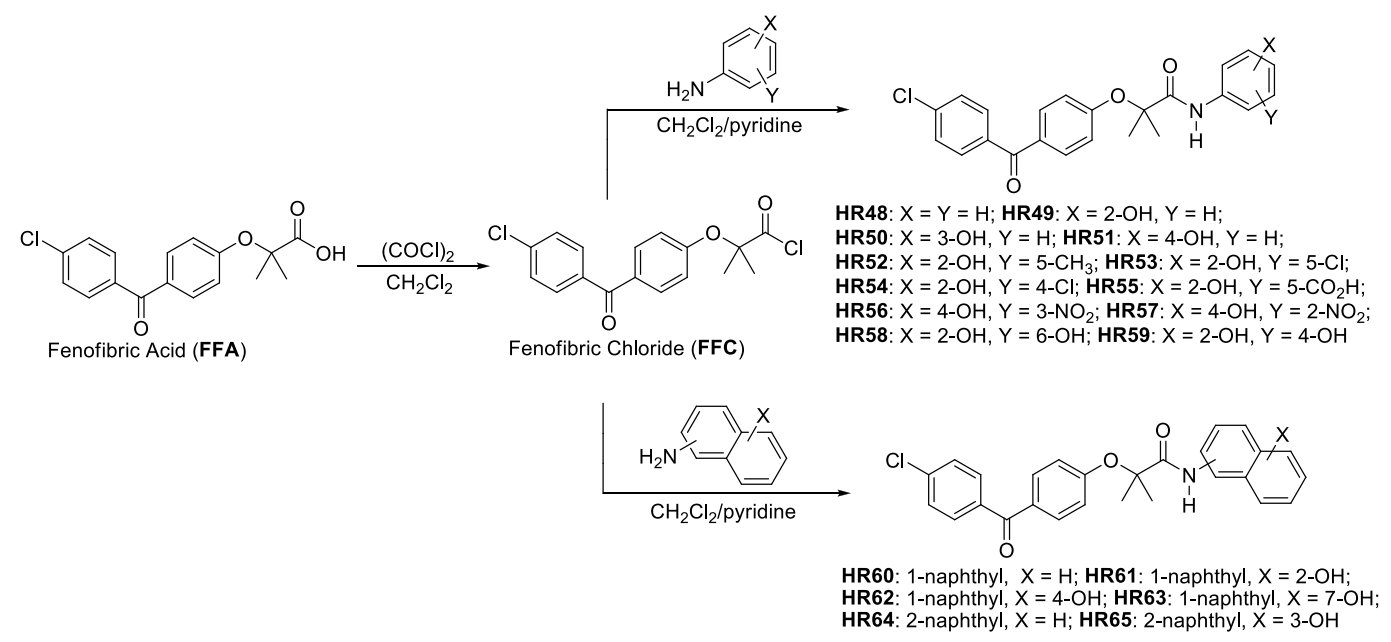

Scheme 3. Schematic illustration of the procedure for preparation of hydroxylated phenyl and naphthyl derivatives of BPA.

Biological, Chemical and Computational Testing of the Compounds: Previously, we have reported that some amide derivatives of FF, including PP1, are more potent than others in eliminating glioblastoma cells ${ }^{26}$ and belong to the large family of BPA ${ }^{39}$. Further modifications of the BPA structure were designed to produce compounds with the improved ability to penetrate BBB, and superior glioblastoma cytotoxicity. This is highly relevant for glioblastoma patients, since one fundamental challenge for new drug development is the need for effective BBB penetration. So far, known BBB-permeable compounds form a very small subset of current oral drugs, and experimental models for testing BBB penetration by new drugs are complex and expensive. Therefore, an independent indicator of the BBB penetration is necessary for faster and more cost effective analyses of new anti-glioblastoma drug candidates. This is critical for the initial 
screening of a large number of compounds, including BPA variants, and selection of best candidates for subsequent measurements of intracranial tumor drug penetration and antitumoral efficacy.

Therefore, we performed extensive molecular modeling prior to preparation of new BPA variants in order to evaluate their specific physicochemical properties considered relevant for the BBB penetration. We have applied first a weighted scoring approach named "Central Nervous System - Multiparameter Optimization" (CNS-MPO) ${ }^{40,41}$. The CNS-MPO algorithm uses 6 key physicochemical properties $(\operatorname{cog} P, \operatorname{cog} \mathrm{D}, \mathrm{MW}, \mathrm{TPSA}$, $\mathrm{HBD}$, and $\mathrm{pKa}$ ) with the scores ranging between 0 and 6.0. Importantly, scores $\geq 4.0$ are widely accepted for selecting compounds with high potential for CNS penetration ${ }^{41}$. The validation of this approach was based on a library of 616 compounds for which experimental distribution of the drug in CNS was determined and the corresponding parameters of the compound incorporated into CNS-MPO scores ${ }^{40,41}$. It was found that CNS-MPO scores of 1-2 (0\%), 2-3 (11.6\% ), 3-4 (40.8\%), $4-5$ (53.8 \%) and 5-6 (81.1\%) correlated with the increased probability of drugs to be found in the brain ${ }^{42}$.

In addition to CNS-MPO, other chemical and physical parameters of the Quantitative Structure Activity Relationship (QSAR) studies were also used in our calculations to further increase the probability of BBB penetration by the compound. These include: molecular polarizability (MP), minimal molecular projection area (MPA), water solubility (LogS), and lipophilicity (cLogP) to name a few. These parameters are not incorporated in the CNS-MPO score, however, they also play a role in the estimation of compound ability to penetrate the BBB ${ }^{43}$. Therefore, we have calculated and incorporated all these additional parameters when evaluating and selecting new HR compounds as 
potential anti-glioblastoma drugs (Figs. 1-6; and supplementary materials). In particular, MP is a response of electron distribution to an externally-applied static electrical field. Comparing MP values helps in understanding how different substrates may change polarization- and dispersion-type during interactions with the active sites of their interacting proteins ${ }^{44}$. It was postulated that MP values between $30-40$ (see supplementary data) are optimal for a molecule to bind to a biotarget ${ }^{45}$. Minimal projected area (MPA) represents another parameter that is important for drug transport and ultimately for drug activity. For instance, a distinct phenotypical pattern of drug recognition and transport for the $\mathrm{G} 616 \mathrm{~N}$ variant was reported, indicating that drug substrates with MPA over $70 \AA^{2}$ are less likely to be transported compared to drugs with smaller MPA ${ }^{46}$. In addition, LogS of -4.5 and greater are indicators of acceptable water solubility ${ }^{47}$, and the rate of passive diffusion is inversely proportionate to the square root of molecular size (Graham's law ${ }^{48}$ ), which are also included in our compound analysis.

Finally, the ability of the compound to penetrate the BBB can also be expressed as a decimal logarithm of brain to plasma concentration ratio $(\log B B)$, which is derived from the modified Clark's equation: $\log B B=0.152$ ClogP $-0.0148 \mathrm{PSA}+0.13930$. It has been shown that compounds with $\log B B>0.3$ readily cross the $\mathrm{BBB}$, while the compounds with $\log \mathrm{BB}<-1.0$ have a problem with the CNS penetration ${ }^{15}$. Therefore, LogBB values for all HR compounds were also calculated and included (Figs. 1-5).

Once all above calculations were performed, initial cell viability $(\mathrm{CV})$ tests were performed for all HR compounds using LN229 human glioblastoma cell line. The cells were treated with proposed HR compounds at $25 \mu \mathrm{M}$, and cell viability was evaluated 
using MTT assay, following a continuous cell exposure to a single dose of the drugs for 72 hrs. (Figs. 1-6).

As a result of this initial screening, we have identified two lead drug candidates, HR51 and HR59, with phenolic moieties that contain BPA structural skeleton similar to our prototype anti-glioblastoma compound PP1 ${ }^{26}$. This is in addition to our recently reported BPA-based compounds (HR28, HR32, HR37, and HR46), which also demonstrated high potential as anti-glioblastoma drugs ${ }^{31}$. Anti-glioblastoma effects of HR51 and HR59 were subsequently confirmed using four different human glioblastoma cell lines, LN229, U-87 MG, U-118 MG, T98G, and the cytotoxicity data were compared to normal human astrocytes (NHA). Results in Fig. 6A demonstrate that all tested glioblastoma cells were partially responsive to $10 \mu \mathrm{M} \mathrm{HR} 51$ and HR59, but were almost completely eliminated following 72 hour exposure to $25 \mu \mathrm{M}$ HR51 or HR59. In contrast, these two compounds were significantly less cytotoxic to normal human astrocytes (NHA), indicating that these two new compounds may have low CNS toxicity. In addition, results in Fig. $6 \mathrm{~B}$ show that $\mathrm{IC}_{50}$ concentrations for HR51 and HR59 are below $10 \mu \mathrm{M}$, which is an acceptable therapeutic concentration for clinically relevant anticancer drugs.

We have also tested if the mechanism of action of HR51 and HR59 is similar to our prototype drugs, PP1 and fenofibrate, which have been previously shown to inhibit mitochondrial respiration at the level of Complex 1 of the electron transport chain $(\mathrm{ETC})^{24,26}$. Indeed, results in Fig. 6C confirmed that both HR51 and HR59 inhibit mitochondrial respiration in the magnitude similar to PP1. 
In addition to a strong in vitro anti-glioblastoma activity (Fig. 6), HR51 and HR59 have physical properties that may contribute to the improved brain tumor penetration. Specifically, HR51 and HR59, have a minimal projection area (MPA) of $46.23 \AA^{2}$ and $43.73 \AA^{2} 4^{6}$, respectively; water solubility (LogS) of -6.61 and $-6.11^{47}$; and brain to plasma concentration ratio (LogBB) of -0.15 and $-0.49^{15}$, which are all considered as highly promising for compounds suspected of being capable of penetrating the brain tumor tissue.

Importantly, HR51 and HR59 can also cross the triple-coculture model of the blood brain barrier (BBB), which consists of astrocytes, pericytes and epithelial cells cultured

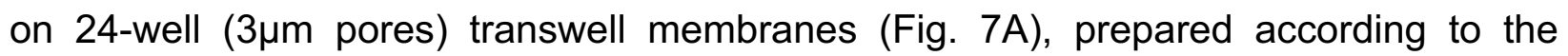
protocol provided by Stone et al. ${ }^{49}$. All experiments in which the ability of HR compounds to cross the BBB were evaluated using the BBB inserts that demonstrated a significant increase of the electric resistance $(\Omega)$ in comparison to inserts without cells ( $\Omega$ BBB $-\Omega$

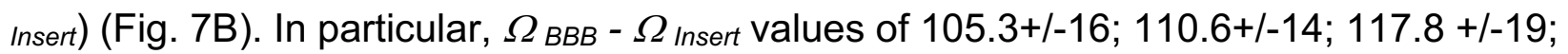
$132.3+/-23$; and $129.0+/-24$ were measured for inserts used to evaluate BBB penetration of FF, caffeine, HR51 and HR59, respectively (Fig. 7B). Accordingly, trans-endothelial electric resistance (TEER $=\Omega$ BBB $-\Omega$ Insert $\mathrm{X}$ area of the membrane) ${ }^{49,50}$ for the inserts used for FF, caffeine, HR51 and HR59 testing are: 34.7+/-5.3; 36.5+/-4.6; 43.7+/-7.6; and 42.6+/-8.1, respectively. These TEER values are comparable to TEER values obtained from similar triple-coculture model of the BBB in which 24-well inserts with $3 \mu \mathrm{m}$ pores were previously evaluated ${ }^{49}$, indicating effective electric resistance produced by our BBB model. 
Following TEER measurements, $25 \mu \mathrm{M} H R 51,25 \mu \mathrm{M}$ HR59, as well as $50 \mu \mathrm{M}$ caffeine (positive control ${ }^{51}$ ) and $25 \mu \mathrm{M}$ fenofibrate (negative control ${ }^{25}$ ) were added to the corresponding insert and the plates were incubated in $37^{\circ} \mathrm{C}$ and $5 \% \mathrm{CO}_{2}$ for $24 \mathrm{hrs}$. Subsequently, aliquots of media from the corresponding inserts and from the wells were collected for HPLC measurements and to calculate BBB permeability $\left(P=V_{A} \cdot C_{A} /\left(t \cdot S \cdot C_{L}\right)\right.$ 52. Results in Fig. 7C show that HR51, HR59 and caffeine cross the in vitro BBB at levels 4.4-fold, 3.5-fold and 22.0-fold higher compared to our internal negative control, fenofibrate, which although has a similar molecular weight and structure to the tested HR compounds (Fig. 1), its ability of crossing natural BBB is very low ${ }^{25}$.

By exploring the computed structural variations of phenolic-BPAs, and testing their cell toxicity, we have demonstrated that the addition of phenol moieties improves antiglioblastoma activity with acceptable LogBB and LogS properties. This could be further improved by adding additional substituent(s) to the phenol moiety, including hydroxy group, halogen, alkyl, nitro on carbonyl to name a few. However, replacing the phenol residue with the larger naphthol, although it might not compromise anti-glioblastoma activity in cell culture, it may result in decreased compound bioavailability mostly due to low water solubility (Fig. 4) ${ }^{53}$. Therefore, these naphthol compounds are less likely to be considered as anti-glioblastoma candidates. Further exploration of phenolic BPAs, using proposed algorithms in combination with the in vitro BBB penetration model, represents a quick, reliable and relatively inexpensive way of testing a large number of new drug candidates in the preparation for extensive pharmacokinetic and efficacy testing in empirical animal models. 
A

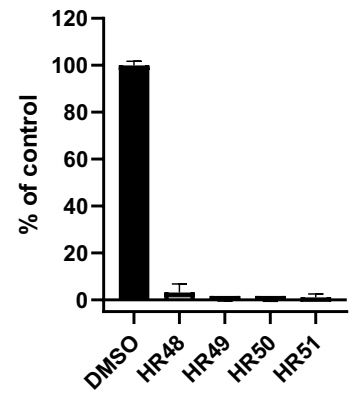

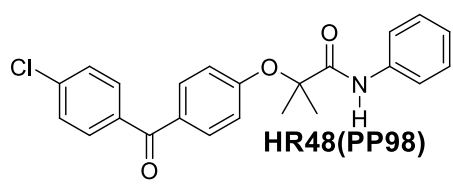

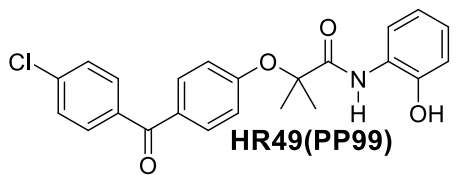<smiles>CC(Oc1ccc(C(=O)c2ccc(Cl)cc2)cc1)(C(=O)Nc1ccccc1)C(=O)Nc1cccc(O)c1</smiles><smiles>CC(Oc1ccc(C(=O)c2ccc(Cl)cc2)cc1)(C(=O)c1ccccc1)C(=O)N(c1ccc(O)cc1)[PH+](P)[PH2+]</smiles>

B

\begin{tabular}{|c|c|c|c|c|c|c|c|c|c|c|}
\hline Comp. & CV & MW & $\begin{array}{c}\text { Clog } \\
\text { P }\end{array}$ & $\begin{array}{c}\text { HB } \\
\text { D }\end{array}$ & HBA & PSA & MPA & logBB & LogS & MPO \\
\hline HR48 & $3.19 \pm 3.64$ & 393.86 & 5.79 & 1 & 3 & 55.40 & 43.73 & 0.20 & -7.07 & 2.59 \\
\hline HR49 & $0 \pm 0.27$ & 409.86 & 5.49 & 2 & 4 & 75.63 & 48.14 & -0.15 & -6.61 & 2.76 \\
\hline HR50 & $0 \pm 0.48$ & 409.86 & 5.49 & 2 & 4 & 75.63 & 43.83 & -0.15 & -6.61 & 2.50 \\
\hline HR51 & $1.1 \pm 1.42$ & 409.86 & 5.49 & 2 & 4 & 75.63 & 46.23 & -0.15 & -6.61 & 2.45 \\
\hline
\end{tabular}

C

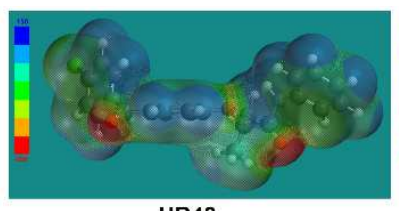

HR48

D

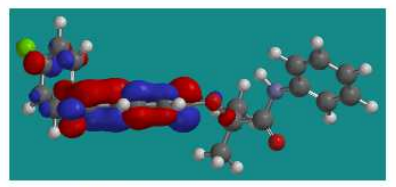

HR48 LUMO (-0.67 eV)

E

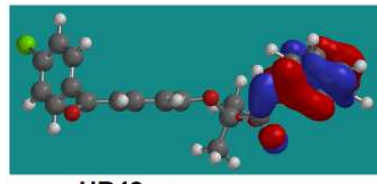

HR48 $($ НОМО $=-9.09 \mathrm{eV})$

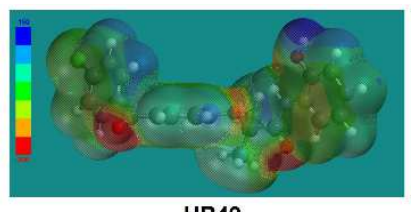

HR49

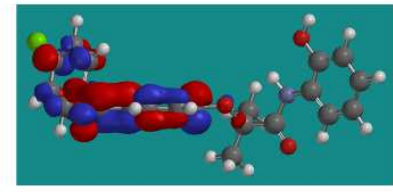

HR49 LUMO (-0.66 eV)

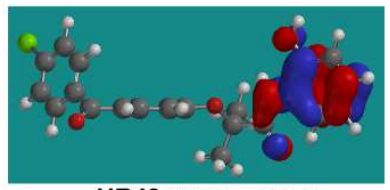

HR49 $(\mathrm{HOMO}=-8.66 \mathrm{eV})$

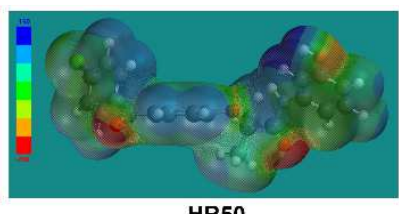

HR50

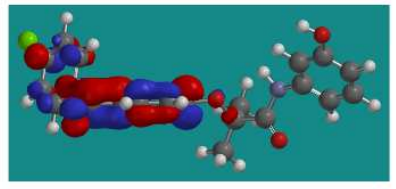

HR50 LUMO (-0.72 eV)

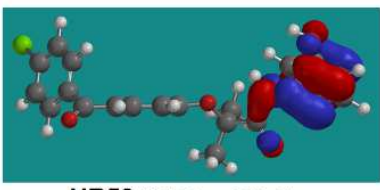

HR50 (HOMO $=-8.96 \mathrm{eV})$

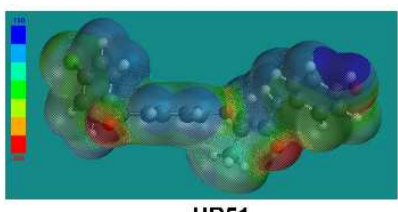

HR51

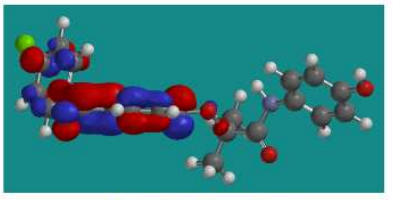

HR51 LUMO $(-0.69 \mathrm{eV})$

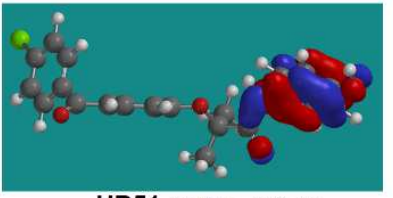

HR51 (HOMO = - $8.81 \mathrm{eV}$ )

Figure 1. Drug candidates with hydroxy substituted phenylamide moiety. Panel A: Cell viability (MTT assay) following exposure to modified variants of $\mathbf{H R 4 8}$ with one hydroxy groups in different positions in the phenylamide moiety ( $25 \mu \mathrm{M}$, for $72 \mathrm{hrs}$ ). Panel B: CV = Cell viability (\% of control) mean $\pm S D$ at 25 $\mu \mathrm{M} ; \mathrm{Clog} P=$ calculated partitioning; $H B D=$ hydrogen bond donor at $p H=7 ; H B A=$ hydrogen bond acceptor at $\mathrm{pH}=7 ; \log B B=$ calculated blood-brain partition; $P S A=$ Polar surface area $\left(\AA^{2}\right) ; M P A=$ Minimal projection area $\left(\AA^{2}\right) ;$ LogS = Aqueous solubility $(\mathrm{mg} / \mathrm{ml}) ; M P O=$ Central nervous system multiparameter optimization (CNS MPO). Panel C: Electrostatic potential map for H48-HR51. Panel D: Computed LUMO orbitals contribution with their energies. Panel E: Computed HOMO orbitals contribution with their energies generated by semi-empirical method PM3 as implemented in Spartan '18 version 1.1.0 

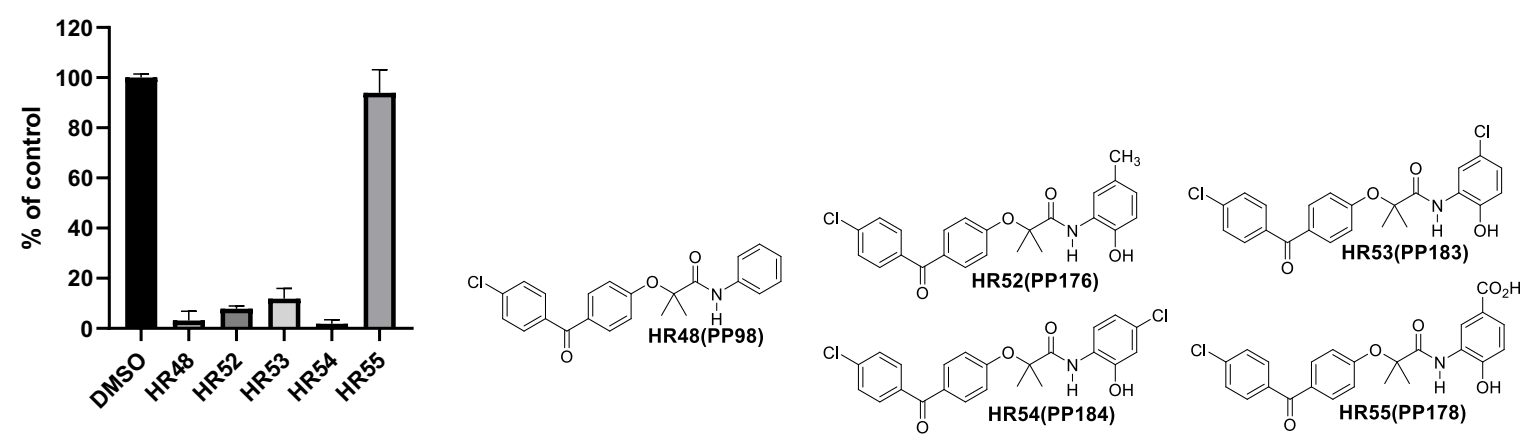

$\mathrm{B}$

\begin{tabular}{|c|c|c|c|c|c|c|c|c|c|c|}
\hline Comp. & CV & MW & ClogP & HBD & HBA & PSA & MPA & logBB & LogS & MPO \\
\hline HR48 & $3.19 \pm 3.64$ & 393.86 & 5.79 & 1 & 3 & 55.40 & 43.73 & 0.20 & -7.07 & 2.59 \\
\hline HR52 & $7.81 \pm 1.12$ & 423.89 & 6.00 & 2 & 4 & 75.63 & 48.18 & -0.07 & -7.09 & 2.49 \\
\hline HR53 & $11.81 \pm 4.17$ & 444.31 & 6.09 & 2 & 4 & 75.63 & 49.87 & -0.05 & -7.10 & 2.79 \\
\hline HR54 & $1.89 \pm 1.56$ & 444.31 & 6.09 & 2 & 4 & 75.63 & 46.41 & -0.05 & -7.08 & 2.90 \\
\hline HR55 & $93.95 \pm 9.16$ & 453.87 & 5.15 & 3 & 6 & 112.93 & 52.16 & -0.75 & -3.20 & 2.72 \\
\hline
\end{tabular}

C

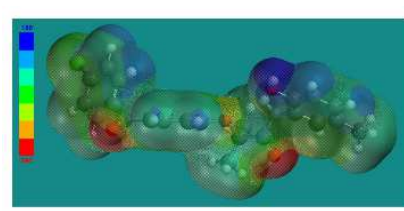

HR52

D

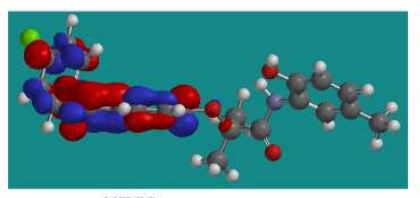

$E$

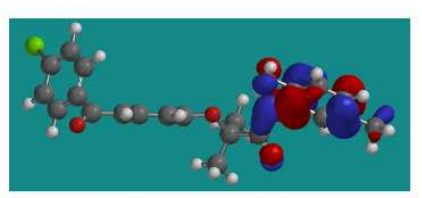

HR52 (HOMO $=-8.87 \mathrm{eV}$ )

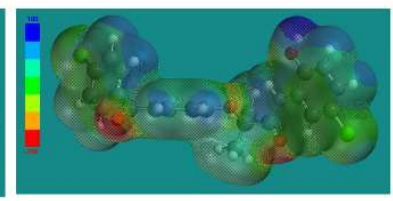

HR53

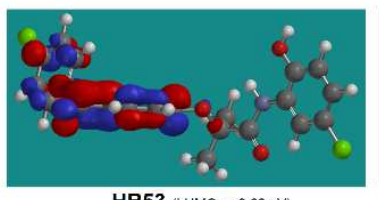

HR53 (LUMO $=-0.69 \mathrm{eV}$ )

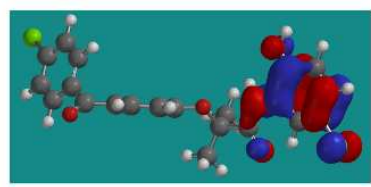

HR53 (HOMO $=-8.70 \mathrm{eV}$ )

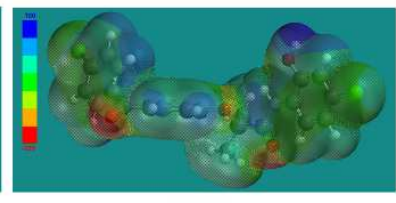

HR54

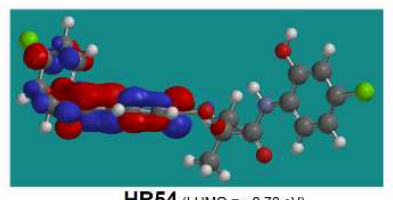

HR54 (LUMO $=-0.70 \mathrm{eV}$ )

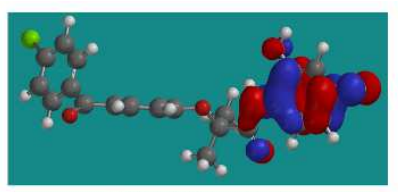

HR54 (HOMO $=-8.66 \mathrm{eV})$

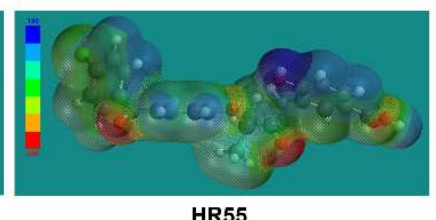

HR55

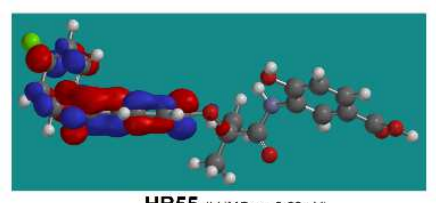

HR55 (LUMO $=-0,69 \mathrm{cV}$ )

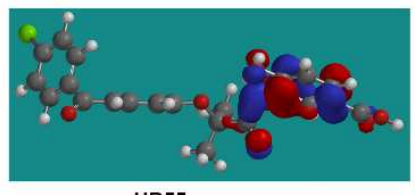

HR55 (HOMO = $-9.32 \mathrm{eV}$ )

Figure 2. Drug candidates with sutituted 2-hydroxyphenylamide moiety. Panel A: Cell viability (MTT assay) following exposure to modified variants of HR48 with ortho hydroxy and ether methyl, chloro, or carboxy group in the phenylamide moiety $(25 \mu \mathrm{M}$, for $72 \mathrm{hrs})$. Panel B: CV = Cell viability (\% of control) mean \pm $S D$ at $25 \mu \mathrm{M} ; \mathrm{Clog} P=$ calculated partitioning; $H B D=$ hydrogen bond donor at $p H=7 ; H B A=$ hydrogen bond acceptor at $\mathrm{pH}=7 ; \log B B=$ calculated blood-brain partition; $P S A=$ Polar surface area $\left(\AA^{2}\right) ; M P A=$ Minimal projection area $\left(\AA^{2}\right) ;$ LogS = Aqueous solubility $(\mathrm{mg} / \mathrm{ml}) ; \mathrm{MPO}=$ Central nervous system multiparameter optimization (CNS MPO). ). Panel C: Electrostatic potential map for H52-HR55. Panel D: Computed LUMO orbitals contribution with their energies. Panel E: Computed HOMO orbitals contribution with their energies generated by semi-empirical method PM3 as implemented in Spartan '18 version 1.1.0 
A
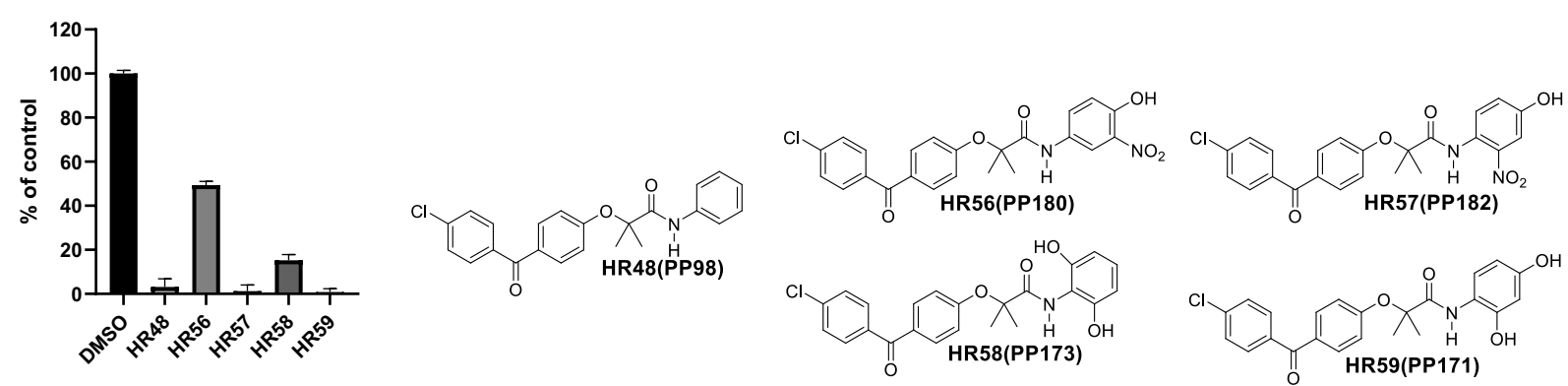

$\mathrm{B}$

\begin{tabular}{|c|c|c|c|c|c|c|c|c|c|c|}
\hline Comp. & CV & MW & ClogP & HBD & HBA & PSA & MPA & logBB & LogS & MPO \\
\hline HR48 & $3.19 \pm 3.64$ & 393.86 & 5.79 & 1 & 3 & 55.40 & 43.73 & 0.20 & -7.07 & 2.59 \\
\hline HR56 & $49.36 \pm 1.67$ & 454.86 & 5.43 & 2 & 6 & $\begin{array}{c}118.7 \\
7\end{array}$ & 47.89 & -0.79 & -6.50 & 1.86 \\
\hline HR57 & $1.29 \pm 2.79$ & 454.86 & 5.43 & 2 & 6 & $\begin{array}{c}118.7 \\
7\end{array}$ & 47.50 & -0.79 & -7.05 & 1.86 \\
\hline HR58 & $15.22 \pm 2.61$ & 425.86 & 5.19 & 3 & 5 & 95.86 & 49.54 & -0.49 & -6.10 & 2.21 \\
\hline HR59 & $0.88 \pm 1.5$ & 425.86 & 5.19 & 3 & 5 & 95.86 & 47.89 & -0.49 & -6.11 & 2.16 \\
\hline
\end{tabular}

C
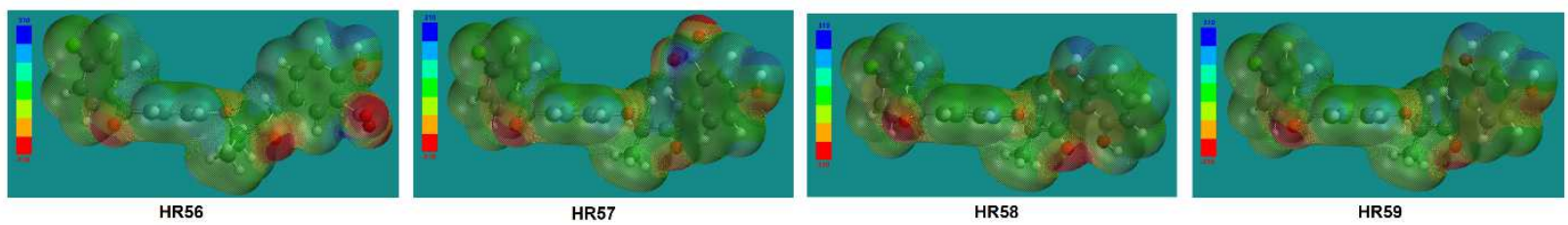

D
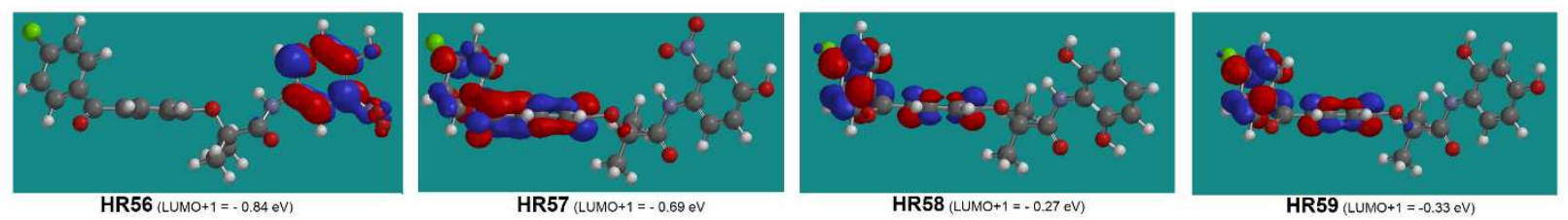

$E$
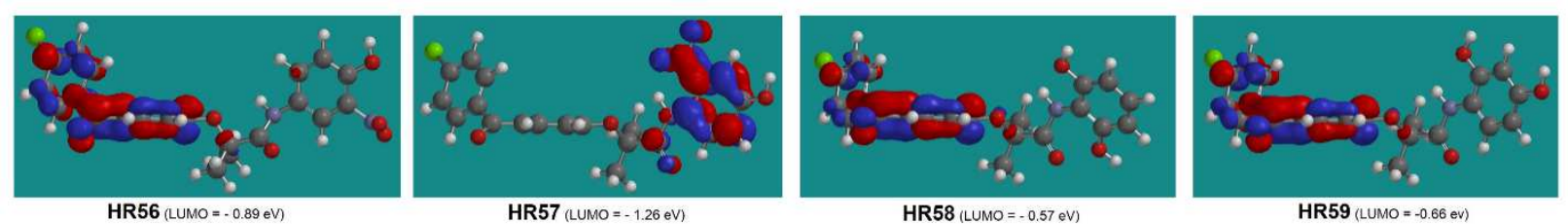

$\mathrm{F}$
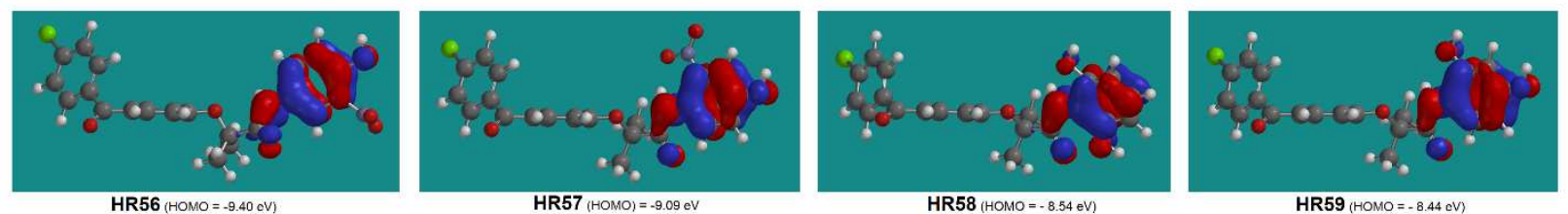

Figure 3. Drug candidates with nitro-hydroxy and two hydroxy sutituted phenylamide moiety. Panel A: Cell viability (MTT assay) following exposure to modified variants of HR48 with one hydroxy and one nitro group or with two hydroxy groups in the phenylamide moiety (25 $\mu \mathrm{M}$, for $72 \mathrm{hrs})$. Panel B: CV = Cell viability (\% of control) mean $\pm S D$ at $25 \mu \mathrm{M}$; ClogP = calculated partitioning; $H B D=$ hydrogen bond donor at $p H=7$; $H B A=$ hydrogen bond acceptor at $p H=7 ; \log B B=$ calculated blood-brain partition; $P S A=$ Polar surface area $\left(\AA^{2}\right) ; M P A=$ Minimal projection area $\left(\AA^{2}\right) ; \log S=$ Aqueous solubility $(\mathrm{mg} / \mathrm{ml}) ; \mathrm{MPO}=$ Central nervous system multiparameter optimization (CNS MPO). Panel C: Electrostatic potential map for H56-HR59. 
Panel D: Computed LUMO+1 orbitals contribution with their energies. Panel E: Computed LUMO orbitals contribution with their energies. Panel F: Computed HOMO orbitals contribution with their energies generated by semi-empirical method PM3 as implemented in Spartan '18 version 1.1.0

A
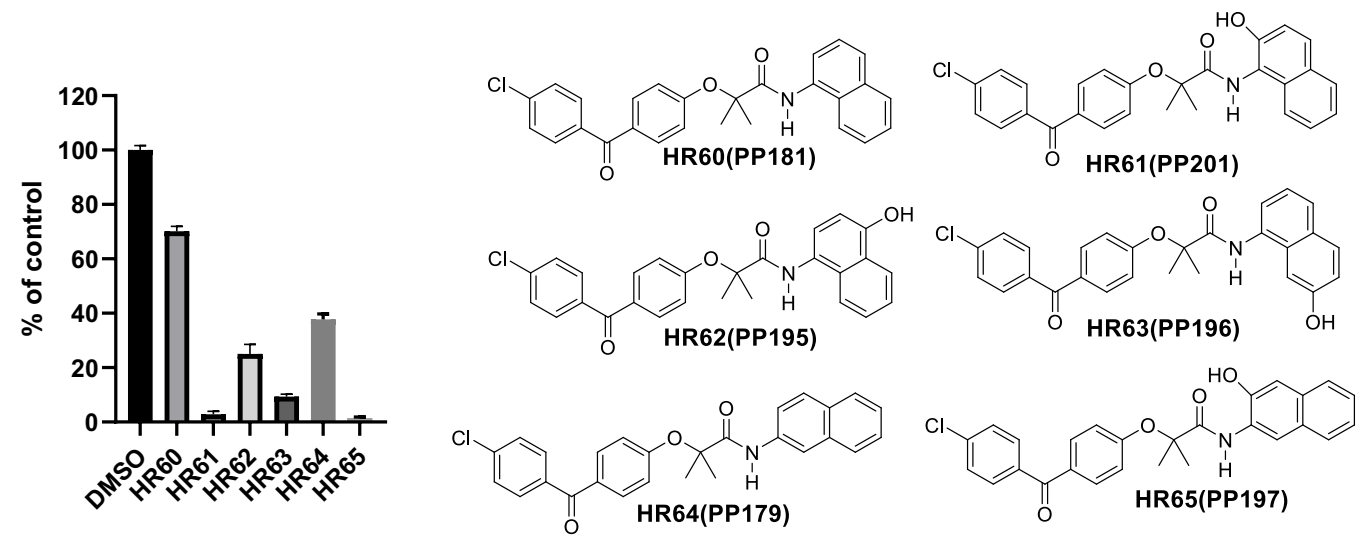

B.

\begin{tabular}{|c|c|c|c|c|c|c|c|c|c|c|}
\hline Comp. & CV & MW & ClogP & $\begin{array}{c}\text { HB } \\
\text { D }\end{array}$ & HBA & PSA & MPA & logBB & LogS & MPO \\
\hline HR60 & $70.13 \pm 1.79$ & 443.92 & 6.78 & 1 & 3 & 55.40 & 50.10 & 0.35 & -8.79 & 2.23 \\
\hline HR61 & $2.87 \pm 1.05$ & 459.92 & 6.48 & 2 & 4 & 75.63 & 48.22 & 0.01 & -8.28 & 2.51 \\
\hline HR62 & $25.03 \pm 3.56$ & 459.92 & 6.48 & 2 & 4 & 75.63 & 50.50 & 0.01 & -8.33 & 2.26 \\
\hline HR63 & $9.33 \pm 0.88$ & 459.92 & 6.48 & 2 & 4 & 75.63 & 53.68 & 0.01 & -8.33 & 1.93 \\
\hline HR64 & $37.7 \pm 2.03$ & 443.92 & 6.78 & 1 & 3 & 55.40 & 50.72 & 0.35 & -8.79 & 2.23 \\
\hline HR65 & $1.49 \pm 0.32$ & 459.92 & 6.48 & 2 & 4 & 75.63 & 50.52 & 0.01 & -8.29 & 2.53 \\
\hline
\end{tabular}

C
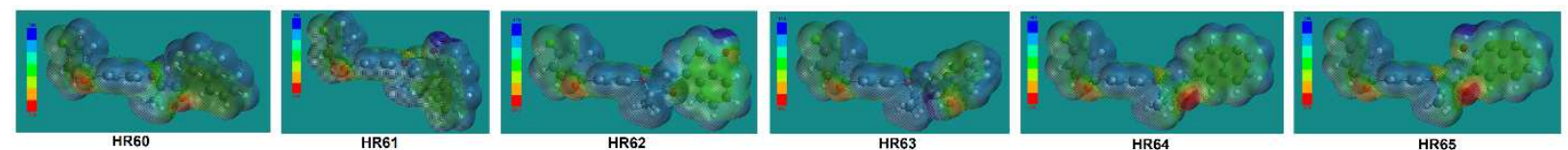

D
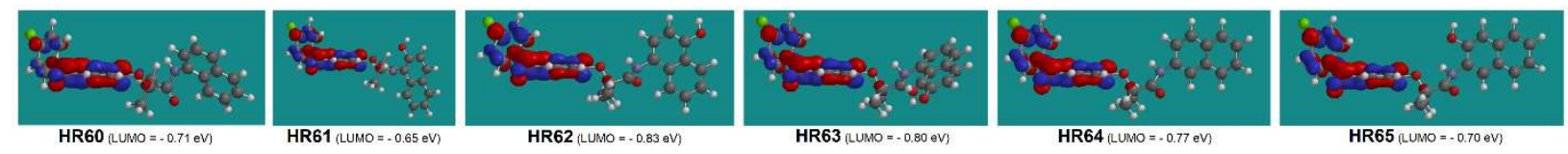

$E$
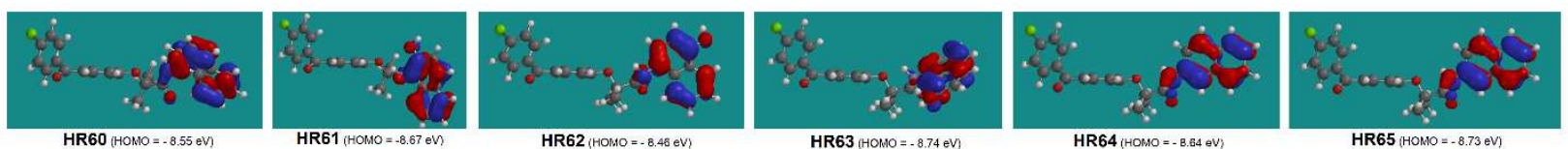

Figure 4. Drug candidates with hydroxy substituted naphthylamide moiety. Panel A: Cell viability (MTT assay) following exposure to modified variants of 1- and 2-naphthylamide of HR60 and HR64 with one hydroxy group in the naphthylamide moiety (25 $\mu \mathrm{M}$, for $72 \mathrm{hrs}$ ). Panel B: CV = Cell viability (\% of control) mean $\pm S D$ at $25 \mu \mathrm{M}$; ClogP = calculated partitioning; $H B D=$ hydrogen bond donor at $p H=7 ; H B A=$ hydrogen bond acceptor at $\mathrm{pH}=7$; $\log B B=$ calculated blood-brain partition; $P S A=$ Polar surface area $\left(\AA^{2}\right)$; $M P A=$ Minimal projection area $\left(\AA^{2}\right) ;$ logS = Aqueous solubility $(\mathrm{mg} / \mathrm{ml}) ; M P O=$ Central nervous system multiparameter optimization (CNS MPO). Panel C: Electrostatic potential map for H60-HR65. Panel D: Computed LUMO orbitals contribution with their energies. Panel E: Computed HOMO orbitals contribution with their energies generated by semi-empirical method PM3 as implemented in Spartan '18 version 1.1.0. 


\begin{tabular}{|c|c|c|c|c|c|c|c|c|c|}
\hline Comp. & logP & PSA & D+A & $\begin{array}{c}\text { M1LogB } \\
\text { B }\end{array}$ & $\begin{array}{c}\text { M2LogB } \\
\text { B }\end{array}$ & $\begin{array}{c}\text { M3LogB } \\
\text { B }\end{array}$ & M4LogBB & logS & $\begin{array}{c}\text { CNS- } \\
\text { MPO }\end{array}$ \\
\hline PP1 & 3.31 & 66.84 & 5 & -0.49 & -0.35 & 0.01 & -1.91 & -5.09 & 3.97 \\
\hline HR48 & 5.79 & 55.40 & 4 & 1.11 & 0.20 & 0.51 & -0.41 & -7.07 & 2.59 \\
\hline HR49 & 5.49 & 75.63 & 6 & 0.39 & -0.15 & 0.26 & -2.27 & -6.61 & 2.76 \\
\hline HR50 & 5.49 & 75.63 & 6 & 0.39 & -0.15 & 0.26 & -2.27 & -6.61 & 2.50 \\
\hline HR51 & 5.49 & 75.63 & 6 & 0.39 & -0.15 & 0.26 & -2.27 & -6.61 & 2.45 \\
\hline HR52 & 6.00 & 75.63 & 6 & 0.65 & -0.07 & 0.34 & -2.15 & -7.09 & 2.49 \\
\hline HR53 & 6.09 & 75.63 & 6 & 0.70 & -0.06 & 0.35 & -2.13 & -7.10 & 2.79 \\
\hline HR54 & 6.09 & 75.63 & 6 & 0.70 & -0.06 & 0.35 & -2.13 & -7.08 & 2.90 \\
\hline HR55 & 5.15 & 112.93 & 9 & -0.81 & -0.75 & -0.17 & -5.26 & -3.20 & 2.74 \\
\hline HR56 & 5.43 & 118.77 & 8 & -0.83 & -0.80 & -0.18 & -4.82 & -6.50 & 1.89 \\
\hline HR57 & 5.43 & 118.77 & 8 & -0.83 & -0.80 & -0.18 & -4.82 & -7.05 & 1.86 \\
\hline HR58 & 5.19 & 95.86 & 8 & -0.32 & -0.49 & 0.01 & -4.13 & -6.10 & 2.21 \\
\hline HR59 & 5.19 & 95.86 & 8 & -0.32 & -0.49 & 0.01 & -4.13 & -6.11 & 2.16 \\
\hline HR60 & 6.78 & 55.40 & 4 & 1.62 & 0.35 & 0.66 & -0.18 & -8.79 & 2.23 \\
\hline HR61 & 6.48 & 75.63 & 6 & 0.90 & 0.002 & 0.41 & -2.04 & -8.28 & 2.51 \\
\hline HR62 & 6.48 & 75.63 & 6 & 0.90 & 0.002 & 0.41 & -2.04 & -8.33 & 2.26 \\
\hline HR63 & 6.48 & 75.63 & 6 & 0.90 & 0.002 & 0.41 & -2.04 & -8.33 & 1.93 \\
\hline HR64 & 6.78 & 55.40 & 4 & 1.62 & 0.35 & 0.66 & -0.18 & -8.79 & 2.23 \\
\hline HR65 & 6.48 & 76.63 & 6 & 0.87 & -0.01 & 0.40 & -2.08 & -8.29 & 2.53 \\
\hline
\end{tabular}

Figure 5. Compiled three variation of computing logBB and estimated MPO-CNS values for HR48 HR65. $\log P$ computed partition, $P S A=$ computed polar surface area; Clog $P=$ calculated partitioning; $P S A=$ Polar surface area $\left(\AA^{2}\right) ; D=$ hydrogen bond donor at $p H=7 ; A=$ hydrogen bond acceptor at $p H$ =7; MPO = Central nervous system multiparameter optimization (CNS MPO).

$M 1 \log B B=0.5159 \times \log P-0.0277 \times P S A-0.3462$. For $\log B B \geq 0.3^{15}$.

$M 2 \log B B=0.152 \log P-0.0148 P S A+0.139$. (Clark's model).

$M 3 \log B B=0.155 \times \log P-0.01 \times P S A+0.164$. (Rishton's model).

$M 4 \log B B=0.2289 \times \log P-0.0326 \times P S A-0.5671 \times(D+A)+2.3420$. For $\log B B \geq-1{ }^{15}$. 
A
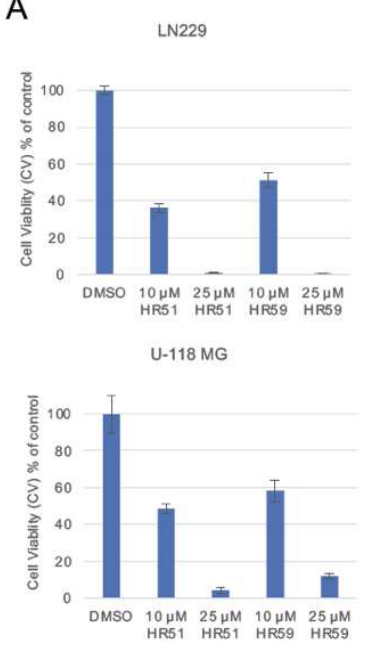

U-87 MG

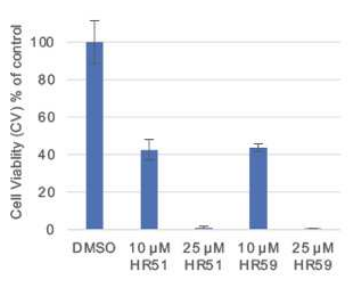

T98G

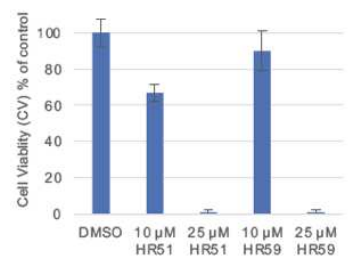

NHA

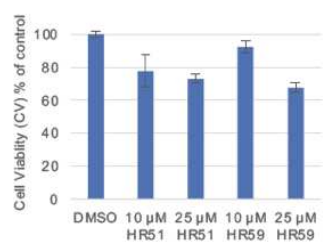

B
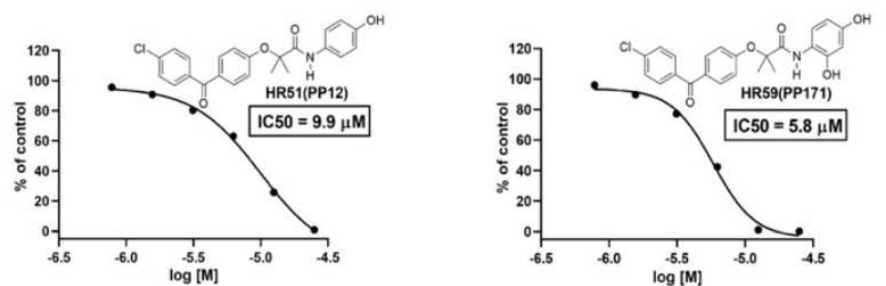

C

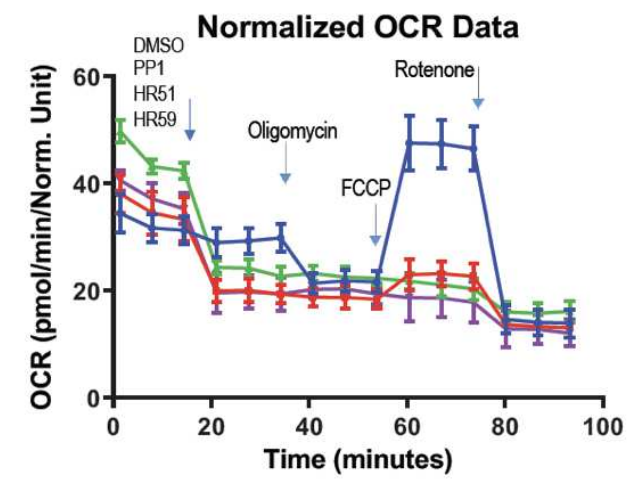

- DMSO

- HR51

- HR59

- PP1

Figure 6. Cytotoxic effects of selected HR compounds. Panel A: Effects of 10 and $25 \mu M$ of HR51 and HR59 on the survival of human glioblastoma cell lines, LN229, U-87MG, U-118 MG, and T98G, compared to the effects of these two compounds on the survival of normal human astrocytes (NHA; LONZA/Clonetics ${ }^{T M}$ ). Data were collected after 72 hours of a continuous cell exposure to a single dose of HR51 or HR59 in the low glucose medium (1g/L). Cells treated with DMSO (vehicle) were used as control. Data are expressed as cell viability (MTT, \% of control) and represent average values with standard deviation $(n=3)$. Panel B: Dose response curves and correlating IC50 values calculated for two most promising HR compounds, HR51 and HR59. Cell viability was evaluated by the MTT assay performed after exposure of LN229 to HR51 and HR59 for $72 \mathrm{hrs}$. Data represent mean values +/- SD ( $n=3)$. Panel C: $\underline{\text { Metabolic }}$ effects of HR51 and HR59 compared to the prototype drug. PP1. Metabolic responses to the drugs were evaluated in LN229 using Extracellular Flux Analyzer XF96 (Seahorse/Agilent). The oxygen consumption rate (OCR; indicative of mitochondrial respiration) was evaluated after injecting DMSO, (negative control), PP1 (positive control) and two experimental drugs, HR51 and HR59, followed by sequential injections of oilgomycin, FCCP; and rotenone (mitochondrial stress assay). Average OCR data were calculated from three independent experiments. Data represent average values +/- SD. Compared to negative control (DMSO), all tested metabolic compounds (PP1, HR51 and HR59) triggered an immediate drop in OCR. In addition, the cells treated with these three compounds did not respond to FCCP injection, indicating loss of the proton gradient across the mitochondrial membrane. 
A

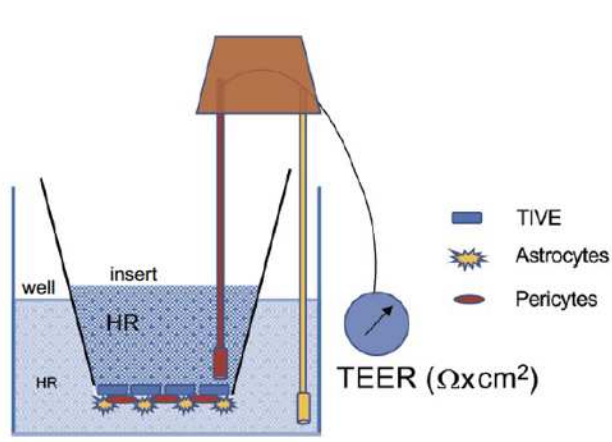

B

\begin{tabular}{|l|l|l|l|l|l|}
\hline & $\begin{array}{l}\text { Fenofibrate } \\
(\mathrm{FF})\end{array}$ & Caffeine & HR49 & HR51 & HR59 \\
\hline TEER $\left(\Omega \times \mathrm{cm}^{2}\right)$ & $\begin{array}{l}34.76+1-5.3 \\
(n=3)\end{array}$ & $\begin{array}{l}36.52+1-4.6 \\
(\mathrm{n}=3)\end{array}$ & $\begin{array}{l}38.9+1-6.4 \\
(\mathrm{n}=3)\end{array}$ & $\begin{array}{l}43.7+1-7.6 \\
(\mathrm{n}=3)\end{array}$ & $\begin{array}{l}42.6+1-8.1 \\
(\mathrm{n}=3)\end{array}$ \\
\hline $\begin{array}{l}\text { TEER } \\
\text { coefficient* }\end{array}$ & 0.95 & 1 & 1.06 & 1.2 & 1.17 \\
\hline$\Omega_{\text {B8B }}-\Omega$ insert & $\begin{array}{l}105.3+1-16 \\
\mathrm{n}=3\end{array}$ & $\begin{array}{l}110.6+1-14 \\
\mathrm{n}=3\end{array}$ & $\begin{array}{l}117.8+1-19 \\
\mathrm{n}=6\end{array}$ & $\begin{array}{l}132.3+1-23 \\
\mathrm{n}=6\end{array}$ & $\begin{array}{l}129.0+1-24 \\
\mathrm{n}=6\end{array}$ \\
\hline
\end{tabular}

*fold change over Caffeine
C

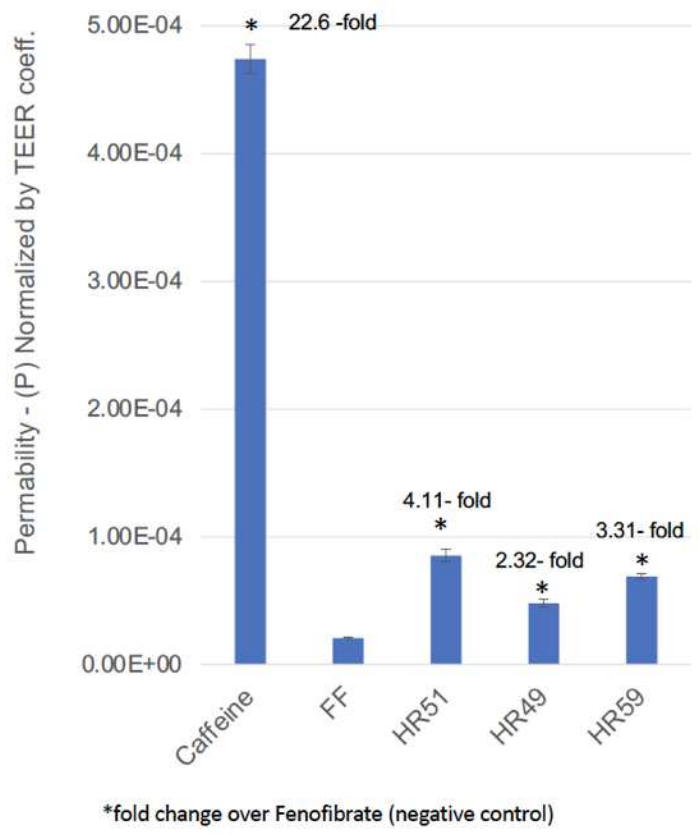

Figure 7: Penetration of the selected HR compounds through in vitro BBB model membrane: Panel A: Schematic representation of a triple-coculture model of the $B B B$, which consists of astrocytes, pericytes and epithelial cells cultured on 24-well transwell membranes with $3 \mu \mathrm{m}$ pores. Trans-endothelial electric resistance (TEER) was measured using a EVOM ${ }^{2}$ meter with a STX3 electrode (World Precision Instruments). Panel B: Measurements of the electric resistance of inserts used for specific compounds ( $\Omega$ ); Difference between resistance of the insert with established BBB versus empty insert ( $\Omega$ BBB $-\Omega$ insert ); and trans-endothelial electric resistance (TEER; $\Omega$ BBB $-\Omega$ insert $/\left(\mathrm{cm}^{2}\right)$. * TEER coefficient $=$ TEER compound $/ T E E R$ caffeine. TEER coefficients were used to normalize BBB permeability $(P)$ values to compensate for differences in TEER values between inserts selected for each compound. Panel C: Differences in $B B B$ permeability $(P)$ calculated using $P=V_{A} \cdot C_{A} /\left(t \cdot S \cdot C_{L}\right)$ equation ${ }^{52}$ and normalized by TEER coefficient. Data represent average values from 2 independent experiments in triplicates $(n=6)$ with standard deviation $S D$. * indicates values significantly different from fenofibrate (internal negative control).

\section{METHODS}


All starting materials were reagent grade and purchased from Sigma-Aldrich, ArkPharm, and TCl America. ${ }^{1} \mathrm{H}-\mathrm{NMR}$ spectra were recorded on Varian Mercury 300 and Varian Mercury 400 Plus instruments in $\mathrm{CDCl}_{3}$ and $\mathrm{DMSO}-\mathrm{d}_{6}$, using the solvent chemical shifts as an internal standard ${ }^{31}$. All computed molecular descriptors were generated by ChemAxon MarvinSketch version 19.20. Electrostatic potential maps were calculated with a PM3 semi-empirical method as implemented in Spartan '18 v 1.1.0 ${ }^{31}$. ${ }^{1} \mathrm{H}-\mathrm{NMR}$ and ${ }^{13} \mathrm{C}-\mathrm{NMR}$ spectra for all HR compounds generated in this study are included in Supplementary Materials.

Method A (Larger scale preparation without extraction or crystallization). 2-(4-(4chlorobenzoyl)phenoxy)-N-(2-hydroxyphenyl)-2-methylpropanamide (HR49). Fenofibric chloride (FFC) was freshly prepared from FFA (4.8 g; $0.015 \mathrm{~mol})$ and oxalyl chloride (2.6 $\mathrm{ml} ; 3.8 \mathrm{~g} ; 0.03 \mathrm{mmol}$ ). After drying with Argon, FFC was dissolved in dichloromethane (30 $\mathrm{ml})$ and mixed with a pyridine $(50 \mathrm{ml})$ solution of 2-aminophenol $(1.3 \mathrm{~g} ; 0.012 \mathrm{~mol})$. The resulting solution was stirred at room temperature for 3 hours, and then at $70^{\circ} \mathrm{C}$ for an additional 3 hours with distillation of dichloromethane. The pyridine was removed under reduced pressure. The resulting solid substance was mixed with ethanol $(50 \mathrm{ml})$ and the resulting mixture was refluxed by stirring until all of the solid was dissolved. This resulting clear alcohol solution was mixed with $3 \%$ sodium carbonate solution $(300 \mathrm{ml})$ and heated with stirring at $80^{\circ} \mathrm{C}$ for one hour. After cooling to room temperature, the insoluble product was separated by filtration, extensively washed with water $(10 \times 100 \mathrm{ml})$ and dried at $110^{\circ} \mathrm{C}$ for 1 hour. The isolated yield was 97\% (4.77 g). ${ }^{1} \mathrm{H}-\mathrm{NMR}$ (DMSO-d, $\left.400 \mathrm{MHz}\right) \delta 9.95$ $(1 \mathrm{H}, \mathrm{s}, \mathrm{OH}), 9.09(1 \mathrm{H}, \mathrm{s}, \mathrm{NH}), 7.93(1 \mathrm{H}, \mathrm{d}, \mathrm{J}=8.0 \mathrm{~Hz}), 7.73(2 \mathrm{H}, \mathrm{d}, \mathrm{J}=8.0 \mathrm{~Hz}), 7.70(2 \mathrm{H}$, 
$\mathrm{d}, \mathrm{J}=8.4 \mathrm{~Hz}), 7.59(2 \mathrm{H}, \mathrm{d}, \mathrm{J}=8.4 \mathrm{~Hz}), 7.11(2 \mathrm{H}, \mathrm{d}, \mathrm{J}=8.4 \mathrm{~Hz}), 6.23(1 \mathrm{H}, \mathrm{t}, \mathrm{J}=7.6 \mathrm{~Hz})$,

$6.83(1 \mathrm{H}, \mathrm{d}, \mathrm{J}=8.0 \mathrm{~Hz}), 6.78(1 \mathrm{H}, \mathrm{t}, \mathrm{J}=8.0 \mathrm{~Hz})$, and $1.60(6 \mathrm{H}, \mathrm{s}) \mathrm{ppm} .{ }^{13} \mathrm{C}-\mathrm{NMR}$ (DMSO-

$\left.d_{6}\right) \delta 193.8,171.7,158.9,147.7,137.6,136.5,132.2,131.7,131.3,129.1,126.1,125.1$,

$121.2,119.9,119.6,115.5,82.3$ and 25.3 ppm.

Method B (small scale preparation). Preparation of 2-(4-(4-chlorobenzoyl)phenoxy)-N(2-hydroxy-5-methylphenyl)-2-methylpropanamide (HR52). A dichloromethane (10 ml) suspension of fenofibric acid (FFA) (191 mg; $0.6 \mathrm{mmol}$ ) and oxalyl chloride $(0.2 \mathrm{ml} ; 384$ $\mathrm{mg} ; 2 \mathrm{mmol}$ ) was stirred at room temperature for five minutes. A few drops of $\mathrm{N}, \mathrm{N}-$ dimethylformamide (DMF) were then added to the suspension, which induced bubbling, and resulted in a clear reaction mixture after approximately 30 minutes. This solution was then stirred at $60^{\circ} \mathrm{C}$ to promote slow solvent evaporation. The solvent residue and oxalyl chloride were removed by drying under an Argon flow at room temperature. The resulting yellow solid substance was dissolved in dichloromethane $(10 \mathrm{ml})$ and mixed with 2-amino4-methylphenol (62 mg; $0.5 \mathrm{mmol})$ in THF (10 ml) and $\mathrm{Na}_{2} \mathrm{CO}_{3}(1.06 \mathrm{~g} ; 10 \mathrm{mmol})$ in water $(10 \mathrm{ml})$. The subsequent mixture was stirred at room temperature for five hours. The solvent was then evaporated under reduced pressure and the residue was mixed with dichloromethane $(50 \mathrm{ml})$ and water $(50 \mathrm{ml})$. This final mixture was sonicated at room temperature until all solid was dissolved. From this bilayer solution, the water layer was discarded, and the dichloromethane layer was washed with $5 \% \mathrm{Na}_{2} \mathrm{CO}_{3}(3 \times 50 \mathrm{ml})$, water (50 ml), $5 \% \mathrm{HCl}(3 \times 50 \mathrm{ml})$, water $(50 \mathrm{ml})$ and dried over anhydrous $\mathrm{Na}_{2} \mathrm{CO}_{3}$. After solvent evaporation, the final product was purified by crystallization from dichloromethane $(\sim 3 \mathrm{ml})$ and hexane $(20 \mathrm{ml})$. The isolated yield was 90\% (190 mg). ${ }^{1} \mathrm{H}-\mathrm{NMR}$ (DMSO-d 6 , $400 \mathrm{MHz}$ ) $\delta 9.74(1 \mathrm{H}$, broad s, OH), $9.06(1 \mathrm{H}, \mathrm{s}, \mathrm{NH}), 7.81(1 \mathrm{H}, \mathrm{s}), 7.73(2 \mathrm{H}, \mathrm{d}, J=8.8 \mathrm{~Hz}), 7.70$ 
$(2 \mathrm{H}, \mathrm{d}, J=8.8 \mathrm{~Hz}), 7.58(2 \mathrm{H}, \mathrm{d}, J=8.8 \mathrm{~Hz}), 7.11(2 \mathrm{H}, \mathrm{d}, J=8.4 \mathrm{~Hz}), 6.73(2 \mathrm{H}, \mathrm{s}), 2.18$ $(3 \mathrm{H}, \mathrm{s})$, and $1.60(6 \mathrm{H}, \mathrm{s}) \mathrm{ppm} .{ }^{13} \mathrm{C}-\mathrm{NMR}\left(\mathrm{DMSO}-\mathrm{d}_{6}\right) \delta$ 193.8, 171.6, 158.8, 145.4, 137.7, $136.5,132.2,131.7,131.3,129.1,128.1,125.9,125.3,121.5,119.9,115.2,82.2,25.3$ and $20.9 \mathrm{ppm}$.

Cell culture and viability assays. All cell culture procedures used for HR compound testing were previously described ${ }^{26,31}$. Briefly, human glioblastoma cell line, LN-229 (ATCC\# CRL-2611), U-87MG (ATCC\# HTB-14), U-118 MG (ATCC\# HTB-15) and T98G (ATCC\# CRL-1690) were maintained as semi-confluent monolayer cultures in DMEM (1 g/L glucose; with sodium pyruvate and L-glutamine) supplemented with $100 \mathrm{U} / \mathrm{ml}$ penicillin, $100 \mu \mathrm{g} / \mathrm{ml}$ streptomycin, and $10 \%$ fetal bovine serum (FBS) at $37^{\circ} \mathrm{C}$ in a $5 \%$ $\mathrm{CO}_{2}$ atmosphere. In addition, normal human astrocytes (NHA) were used to evaluate effects of the selected HR compounds may have on normal not transformed cells. NHA were cultured according to the manufacturer protocol (LONZA/Clonetics ${ }^{\mathrm{TM}}$ ). Prior to the treatment with HR compounds, the cells were plated in 96-well plates (BD Falcon) at the initial density of $2 \times 10^{4} /$ well. Twenty-four hours after plating, stock solutions of the HR compounds were prepared in DMSO, diluted in cell culture medium, to the final concentration of $25 \mu \mathrm{M}$, and added to the cells in triplicate for every experimental condition. For the vehicle control, DMSO was used at $0.5 \%$. After $72 \mathrm{~h}$ incubation, MTT assay (surrogate for cell viability) was performed according to our previous publications ${ }^{31}$. Briefly, following $1.5 \mathrm{~h}$ incubation with MTT, formazan crystals were dissolved in $5 \mathrm{mM}$ $\mathrm{HCl}$ in isopropanol and the absorbance read at $540 \mathrm{~nm}$. Data represent mean values expressed as $\%$ cell viability of control $(\mathrm{DMSO}) \pm \mathrm{SD}$. Phase contrast images of treated 
cells were taken 72 hours after treatment with HR compounds with a BZ-X800 fluorescence microscope (Keyence) equipped with a 20x objective. The drug dose causing $50 \%$ inhibition in the MTT assay at 72 hour time point (half maximal inhibitory concentration - IC50) was calculated using GraphPad Prism 8.

In vitro model of the blood brain barrier (BBB). The blood brain barrier was re-created in vitro using a modified protocol provided by Stone et al ${ }^{49}$. Briefly, 24-well transwell inserts (Falcon, catalog number 353096) were coated with $10 \mu \mathrm{g} / \mathrm{cm}^{2}$ of Collagen Type IV from human placentas (Sigma) for $24 \mathrm{hrs}$ at $4^{\circ} \mathrm{C}$. Inserts were washed with sterile water and air-dried for $2 \mathrm{hrs}$. Next, the inserts were coated with $2 \mu \mathrm{g} / \mathrm{cm}^{2}$ poly-L-lysine (ScienCell) for $1 \mathrm{hr}$ at $37^{\circ} \mathrm{C}$, then washed twice with sterile $\mathrm{H}_{2} \mathrm{O}$ and air-dried for $2 \mathrm{hrs}$. $1.57 \times 10^{5}$ primary human astrocytes (ScienCell) and $3.125 \times 10^{4}$ primary human pericytes (ScienCell) were resuspended in $25 \mu \mathrm{l}$ of astrocyte medium and pericyte medium (ScienCell), respectively, then combined in a 1:1 ratio for $50 \mu$ l total volume. Dried, coated inserts were turned upside down such that the basolateral surface was exposed at the top, and $50 \mu$ l of the cell mixture was added to the membrane, covered with the plate lid, and incubated for $2 \mathrm{hrs}$ at $37^{\circ} \mathrm{C}$ to allow cell adherence. Any medium remaining on top of the membrane was carefully removed before returning inserts to their upright position with the apical surface facing upward as they were placed in a 24-well plate containing $500 \mu \mathrm{l}$ per well of astrocyte/pericyte medium (1:1). An additional $300 \mu \mathrm{L}$ of medium was added to the apical compartment. Four days after plating, the apical compartment medium was removed, and $3.75 \times 10^{4}$ of telomerase-immortalized vain endothelial cells (TIVE; provided by Dr. Rolf Renne) in $50 \mu$ l of TIVE medium ${ }^{54}$ were added and incubated 
for $5 \mathrm{hrs}$ at $37^{\circ} \mathrm{C}$ to allow cell adherence, followed by the addition of an extra $250 \mu \mathrm{l}$ of TIVE medium. Half the volume of the corresponding media in the lower and upper compartment was replaced with fresh media every third day. Ten days after initial plating, trans-endothelial electric resistance (TEER) was measured using a EVOM ${ }^{2}$ meter with a STX3 electrode (World Precision Instruments). The ability of selected HR compounds to pass through in vitro BBB was tested using inserts with effectively reconstructed BBB as confirmed by TEER ${ }^{49,50}$.

HPLC detection of selected HR compounds: Following TEER measurement, the medium from apical compartment of the in vitro BBB model (Fig. 7A) was replaced with $350 \mu \mathrm{L}$ of fresh TIVE medium containing corresponding compounds [HR49 (PP99), HR51 (PP12), HR59 (PP171)] all used at 25mM. In addition, 25mM fenofibrate (FF), which does not cross the BBB ${ }^{25}$ was used as negative control, and $50 \mu \mathrm{M}$ caffeine was used as a positive control ${ }^{51}$. Plates containing the inserts were returned to the incubator $\left(37^{\circ} \mathrm{C}, 5 \%\right.$ $\mathrm{CO}_{2}$ ), and after $24 \mathrm{hrs}$ of incubation conditioned media from basolateral (well) and apical (insert) compartments were collected and frozen for quantitative analyses by HPLC. $100 \mu l$ aliquots of the collected samples were subsequently mixed with $100 \mu$ of $100 \%$ acetonitrile, samples were centrifuged at $16.000 \mathrm{rpm}$ at $4{ }^{\circ} \mathrm{C}$ for $10 \mathrm{~min}$ and supernatants collected for HPLC analyses using UltiMate 3000 system (Thermo Scientific) equipped

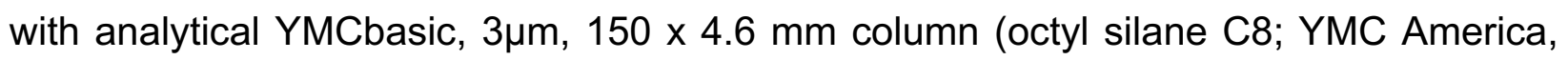
Inc.). Isocratic elution of the compounds was performed using mobile phase composed of solvent $\mathrm{A}$ ( $50 \mathrm{mM}$ acetic acid in $\mathrm{dH}_{2} \mathrm{O}$ ) and solvent $\mathrm{B}$ (acetonitrile) mixed at ratios predetermined for each compound (Table 1). All separations were carried out with sample 
volume of $5 \mu \mathrm{l}$ at flow rate of $1 \mathrm{ml} / \mathrm{min}$, at $20^{\circ} \mathrm{C}$. Concentration of each compound was calculated using serial dilutions of the known concentration of the compound separated at the same run with experimental and control samples. After separation, integrated areas under the peak were used to prepare calibration curves and to determine concentration of the compounds.

Table1. Details of the HPLC method for selected HR compounds.

\begin{tabular}{|l|l|l|l|l|}
\hline Compound & Method length [min] & Concentration solvent B [\%] & Detection wavelength [nm] & Retention time [min] \\
\hline Caffeine & 5 & 25 & 272 & 2.54 \\
\hline Fenofibrate & 10 & 70 & 288 & 5.84 \\
\hline HR51 & 6 & 60 & 260 & 4.3 \\
\hline HR59 & 5 & 60 & 262 & 3.92 \\
\hline
\end{tabular}

\section{Evaluation of metabolic parameters}

Metabolic responses of human glioblastoma cells were evaluated with Extracellular Flux Analyzer XFe96 (Agilent Technologies). During the day prior to each assay the cells were plated at $2 \times 10^{4}$ cells/well in Agilent Seahorse 96-well XF cell culture microplates with growth supporting media and incubated overnight. At the time of measurement, growth media were replaced with serum-free XF assay medium (Seahorse XF Base Medium supplemented with $1 \mathrm{mM}$ sodium pyruvate, $2 \mathrm{mM}$ glutamine, and 5.5 $\mathrm{mM}$ glucose) and cartridges equipped with oxygen-sensitive and $\mathrm{pH}$-sensitive fluorescent probes were placed above the cells. The oxygen consumption rate (OCR; indicative of mitochondrial respiration) was evaluated after injecting HR compounds or PP1 (all used at $25 \mu \mathrm{M})$, or DMSO $(0.1 \%$; vehicle control). These initial injections were followed by sequential injections of metabolic toxins to execute mitochondrial stress assay: oligomycin (inhibitor of ATP synthase; $0.5 \mu \mu \mathrm{M}$ ); carbonylcyanide-p- 
trifluoromethoxyphenylhydrazone (FCCP; uncoupling factor; $0.5 \mu \mathrm{M}$ ), rotenone (inhibitor of mitochondrial complex I; $0.3 \mu \mathrm{M}$ ), and antimycin A (inhibitor of mitochondrial complex III; $0.3 \mu \mathrm{M})$. 


\section{References}

1 Nakada, M. et al. Molecular targets of glioma invasion. Cell Mol Life Sci 64, 458478 (2007).

2 Terzis, A. J., Niclou, S. P., Rajcevic, U., Danzeisen, C. \& Bjerkvig, R. Cell therapies for glioblastoma. Expert opinion on biological therapy 6, 739-749 (2006).

3 Montemurro, N. Glioblastoma Multiforme and Genetic Mutations: The Issue Is Not Over Yet. An Overview of the Current Literature. J Neurol Surg A Cent Eur Neurosurg 81, 64-70, doi:10.1055/s-0039-1688911 (2020).

4 Brennan, C. W. et al. The somatic genomic landscape of glioblastoma. Cell 155, 462-477, doi:10.1016/j.cell.2013.09.034 (2013).

5 Network, T. C. Corrigendum: Comprehensive genomic characterization defines human glioblastoma genes and core pathways. Nature 494, 506, doi:10.1038/nature11903 (2013).

6 Wu, F. et al. Molecular classification of IDH-mutant glioblastomas based on gene expression profiles. Carcinogenesis, doi:10.1093/carcin/bgz032 (2019).

7 Bagley, S. J. \& O'Rourke, D. M. Clinical investigation of CAR T cells for solid tumors: Lessons learned and future directions. Pharmacol Ther, 107419, doi:10.1016/j.pharmthera.2019.107419 (2019).

8 Romani, M., Pistillo, M. P., Carosio, R., Morabito, A. \& Banelli, B. Immune Checkpoints and Innovative Therapies in Glioblastoma. Frontiers in oncology 8, 464, doi:10.3389/fonc.2018.00464 (2018).

9 Alifieris, C. \& Trafalis, D. T. Glioblastoma multiforme: Pathogenesis and treatment. Pharmacol Ther 152, 63-82, doi:10.1016/j.pharmthera.2015.05.005 (2015).

10 Perry, A. \& Wesseling, P. Histologic classification of gliomas. Handb Clin Neurol 134, 71-95, doi:10.1016/B978-0-12-802997-8.00005-0 (2016).

11 Rulseh, A. M. \& Vymazal, J. Whole brain apparent diffusion coefficient measurements correlate with survival in glioblastoma patients. Journal of neurooncology 146, 157-162, doi:10.1007/s11060-019-03357-y (2020).

12 Biasibetti, E. et al. Comparison of Allogeneic and Syngeneic Rat Glioma Models by Using MRI and Histopathologic Evaluation. Comp Med 67, 147-156 (2017).

13 Harder, B. G. et al. Developments in Blood-Brain Barrier Penetrance and Drug Repurposing for Improved Treatment of Glioblastoma. Frontiers in oncology 8 , 462, doi:10.3389/fonc.2018.00462 (2018).

14 Ostermann, S. et al. Plasma and cerebrospinal fluid population pharmacokinetics of temozolomide in malignant glioma patients. Clin Cancer Res 10, 3728-3736, doi:10.1158/1078-0432.CCR-03-0807 (2004).

15 Vilar, S., Chakrabarti, M. \& Costanzi, S. Prediction of passive blood-brain partitioning: Straightforward and effective classification models based on in silico derived physicochemical descriptors. J Mol Graph Model 28, 899-903, doi:10.1016/j.jmgm.2010.03.010 (2010). 
16 Kitange, G. J. et al. Induction of MGMT expression is associated with temozolomide resistance in glioblastoma xenografts. Neuro Oncol 11, 281-291, doi:10.1215/15228517-2008-090 (2009).

17 Gao, L. et al. Suppression of glioblastoma by a drug cocktail reprogramming tumor cells into neuronal like cells. Sci Rep 9, 3462, doi:10.1038/s41598-01939852-5 (2019).

18 Lu, G. et al. Triple-drug Therapy With Bevacizumab, Irinotecan, and Temozolomide Plus Tumor Treating Fields for Recurrent Glioblastoma: A Retrospective Study. Front Neurol 10, 42, doi:10.3389/fneur.2019.00042 (2019).

19 Maraka, S. et al. Phase 1 lead-in to a phase 2 factorial study of temozolomide plus memantine, mefloquine, and metformin as postradiation adjuvant therapy for newly diagnosed glioblastoma. Cancer 125, 424-433, doi:10.1002/cncr.31811 (2019).

20 Drukala, J. et al. ROS accumulation and IGF-IR inhibition contribute to fenofibrate/PPARalpha -mediated inhibition of glioma cell motility in vitro. $\mathrm{Mol}$ Cancer 9, 159, doi:1476-4598-9-159 [pii]

10.1186/1476-4598-9-159 (2010).

21 Grabacka, M. M. et al. Fenofibrate Induces Ketone Body Production in Melanoma and Glioblastoma Cells. Frontiers in endocrinology 7, 5, doi:10.3389/fendo.2016.00005 (2016).

22 Koltai, T. Fenofibrate in cancer:mechanisms involved in anticancer activity. F1000Research 4, 1-22, doi:10.12688/f1000research.6153.1 (2015).

23 Wybieralska, E. et al. Fenofibrate attenuates contact-stimulated cell motility and gap junctional coupling in DU-145 human prostate cancer cell populations. Oncol Rep 26, 447-453, doi:10.3892/or.2011.1321 (2011).

24 Wilk, A. et al. Molecular Mechanisms of Fenofibrate-Induced Metabolic Catastrophe and Glioblastoma Cell Death. Molecular and cellular biology, doi:10.1128/MCB.00562-14 (2014).

25 Grabacka, M. et al. Fenofibrate subcellular distribution as a rationale for the intracranial delivery through biodegradable carrier. Journal of physiology and pharmacology : an official journal of the Polish Physiological Society 66, 233-247 (2015).

26 Stalinska, J. et al. Chemically Modified Variants of Fenofibrate with Antiglioblastoma Potential. Trans/ Oncol 12, 895-907, doi:10.1016/j.tranon.2019.04.006 (2019).

27 Abotaleb, M. et al. Flavonoids in Cancer and Apoptosis. Cancers 11, doi:10.3390/cancers11010028 (2018).

28 Doan, P. et al. Alkylaminophenol Induces G1/S Phase Cell Cycle Arrest in Glioblastoma Cells Through p53 and Cyclin-Dependent Kinase Signaling Pathway. Front Pharmacol 10, 330, doi:10.3389/fphar.2019.00330 (2019).

29 Rao, S., Chinkwo, K., Santhakumar, A., Johnson, S. \& Blanchard, C. Apoptosis Induction Pathway in Human Colorectal Cancer Cell Line SW480 Exposed to Cereal Phenolic Extracts. Molecules 24, doi:10.3390/molecules24132465 (2019).

30 Romanos-Nanclares, A. et al. Phenolic Acid Subclasses, Individual Compounds, and Breast Cancer Risk in a Mediterranean Cohort: The SUN Project. J Acad Nutr Diet, doi:10.1016/j.jand.2019.11.007 (2020). 
31 Stalinska, J. et al. Exploring anticancer activity of structurally modified benzylphenoxyacetamide (BPA); I: Synthesis strategies and computational analyses of substituted BPA variants with high anti-glioblastoma potential. Sci Rep 9, 17021, doi:10.1038/s41598-019-53207-0 (2019).

32 Prinz, H. How to identify a pharmacophore. Chem Biol 15, 207-208, doi:10.1016/j.chembiol.2008.02.013 (2008).

33 Zeslawska, E. et al. Pharmacophoric features for a very potent 5spirofluorenehydantoin inhibitor of cancer efflux pump ABCB1, based on X-ray analysis. Chem Biol Drug Des 93, 844-853, doi:10.1111/cbdd.13473 (2019).

34 Dzialo, M. et al. The Potential of Plant Phenolics in Prevention and Therapy of Skin Disorders. Int J Mol Sci 17, 160, doi:10.3390/ijms17020160 (2016).

35 Brown, D. G. \& Bostrom, J. Analysis of Past and Present Synthetic Methodologies on Medicinal Chemistry: Where Have All the New Reactions Gone? J Med Chem 59, 4443-4458, doi:10.1021/acs.jmedchem.5b01409 (2016).

36 Pattabiraman, V. R. \& Bode, J. W. Rethinking amide bond synthesis. Nature 480, 471-479, doi:10.1038/nature10702 (2011).

37 Dunetz, J. R., Magano, J. \& Weisenburger, G. A. Large-Scale Applications of Amide Coupling Reagents for the Synthesis of Pharmaceuticals. Org Process Res Dev 20, 140-177, doi:10.1021/op500305s (2016).

38 Greenberg, J. A. \& Sammakia, T. The Conversion of tert-Butyl Esters to Acid Chlorides Using Thionyl Chloride. J Org Chem 82, 3245-3251, doi:10.1021/acs.joc.6b02931 (2017).

39 Rani, P., Pal, D., Hegde, R. R. \& Hashim, S. R. Anticancer, Anti-Inflammatory, and Analgesic Activities of Synthesized 2-(Substituted phenoxy) Acetamide Derivatives. Biomed Res Int, doi:Artn 386473

$10.1155 / 2014 / 386473$ (2014).

40 Wager, T. T., Hou, X. J., Verhoest, P. R. \& Villalobos, A. Moving beyond Rules: The Development of a Central Nervous System Multiparameter Optimization (CNS MPO) Approach To Enable Alignment of Druglike Properties. Acs Chem Neurosci 1, 435-449, doi:10.1021/cn100008c (2010).

41 Wager, T. T., Hou, X. J., Verhoest, P. R. \& Villalobos, A. Central Nervous System Multiparameter Optimization Desirability: Application in Drug Discovery. Acs Chem Neurosci 7, 767-775, doi:10.1021/acschemneuro.6b00029 (2016).

42 Rankovic, Z. CNS Drug Design: Balancing Physicochemical Properties for Optimal Brain Exposure. J Med Chem 58, 2584-2608, doi:10.1021/jm501535r (2015).

43 Wu, Z. Y. et al. Comparison of prediction models for blood brain barrier permeability and analysis of the molecular descriptors. Pharmazie 67, 628-634 (2012).

44 Wang, J. M. et al. Development of Polarizable Models for Molecular Mechanical Calculations I: Parameterization of Atomic Polarizability. J Phys Chem B 115, 3091-3099, doi:10.1021/jp112133g (2011).

45 Naef, R. A Generally Applicable Computer Algorithm Based on the Group Additivity Method for the Calculation of Seven Molecular Descriptors: Heat of Combustion, LogPO/W, LogS, Refractivity, Polarizability, Toxicity and LogBB of 
Organic Compounds; Scope and Limits of Applicability. Molecules 20, 1827918351, doi:10.3390/molecules201018279 (2015).

46 Cha, H. J., Muller, R. T. \& Pos, K. M. Switch-Loop Flexibility Affects Transport of Large Drugs by the Promiscuous AcrB Multidrug Efflux Transporter. Antimicrob Agents Ch 58, 4767-4772, doi:10.1128/Aac.02733-13 (2014).

47 Savjani, K. T., Gajjar, A. K. \& Savjani, J. K. Drug solubility: importance and enhancement techniques. ISRN Pharm 2012, 195727, doi:10.5402/2012/195727 (2012).

48 Piiper, J. \& Worth, H. Value and limits of Graham's law for prediction of diffusivities of gases in gas mixtures. Respir Physiol 41, 233-240 (1980).

49 Stone, N. L., England, T. J. \& O'Sullivan, S. E. A Novel Transwell Blood Brain Barrier Model Using Primary Human Cells. Front Cell Neurosci 13, 230, doi:10.3389/fncel.2019.00230 (2019).

50 Srinivasan, B. et al. TEER measurement techniques for in vitro barrier model systems. J Lab Autom 20, 107-126, doi:10.1177/2211068214561025 (2015).

51 Garberg, P. et al. In vitro models for the blood-brain barrier. Toxicol In Vitro 19, 299-334, doi:10.1016/j.tiv.2004.06.011 (2005).

52 Wang, Y. et al. An experimentally validated approach to calculate the blood-brain barrier permeability of small molecules. Sci Rep 9, 6117, doi:10.1038/s41598019-42272-0 (2019).

53 Dahan, A. \& Miller, J. M. The solubility-permeability interplay and its implications in formulation design and development for poorly soluble drugs. AAPS J 14, 244251, doi:10.1208/s12248-012-9337-6 (2012).

54 An, F. Q. et al. Long-term-infected telomerase-immortalized endothelial cells: a model for Kaposi's sarcoma-associated herpesvirus latency in vitro and in vivo. $J$ Virol 80, 4833-4846, doi:10.1128/JVI.80.10.4833-4846.2006 (2006). 
ACKNOWLEDGEMENTS: This work was supported by P20-GM121288-01 (KR) and matching funds from the Dean, School of Medicine, LSU Health Sciences Center (KR). All chemistry and computational studies were supported by STEPFARM, LLC. (BSJ).

\section{AUTHOR INFORMATION}

Joanna Stalinska, MS

Neurological Cancer Research, Stanley S. Scott Cancer Center, Department of Interdisciplinary Oncology, LSU Health Sciences Center, New Orleans, LA 70112; and Department of Cell Biology, Faculty of Biochemistry, Biophysics and Biotechnology, Jagiellonian University, Cracow Poland.

Charles $\mathrm{H}$. Ingraham IV

Neurological Cancer Research, Stanley S. Scott Cancer Center, Department of Interdisciplinary Oncology, LSU Health Sciences Center, New Orleans, LA 70112D Sean C. Carson. MS

Department of Chemistry, University of New Orleans, New Orleans, LA 70148, United States.

Cecilia Vittori, MS

Neurological Cancer Research, Stanley S. Scott Cancer Center, Department of Interdisciplinary Oncology, LSU Health Sciences Center, New Orleans, LA 70112; and Department of Biomedical and Clinical Sciences L. Sacco, University of Milan, Milan, Italy.

Adam Lassak, PhD

Neurological Cancer Research, Stanley S. Scott Cancer Center, Department of Interdisciplinary Oncology, LSU Health Sciences Center, New Orleans, LA 70112 Karlie Bonstaff, PhD

Neurological Cancer Research, Stanley S. Scott Cancer Center, Department of Interdisciplinary Oncology, LSU Health Sciences Center, New Orleans, LA 70112 Celeste Faia, PhD student Neurological Cancer Research, Stanley S. Scott Cancer Center, Department of Interdisciplinary Oncology, LSU Health Sciences Center, New Orleans, LA 70112 Francesca Peruzzi, PhD Neurological Cancer Research, Stanley S. Scott Cancer Center, Department of Interdisciplinary Oncology, LSU Health Sciences Center, New Orleans, LA 70112 Susan Colley, MS

Grants and Development Office, Stanley S. Scott Cancer Center, LSU Health Sciences Center, New Orleans, LA 70112.

\section{Corresponding Authors}

Krzysztof Reiss, PhD

Neurological Cancer Research, Stanley S. Scott Cancer Center, Department of Interdisciplinary Oncology, LSU Health Sciences Center, New Orleans, LA 70112; email: kreiss@lsuhsc.edu; tel: 5042102977.

and

Branko S. Jursic, PhD 
Department of Chemistry, University of New Orleans, New Orleans, LA 70148, United States and Stepharm Ilc., PO Box 24220, New Orleans, LA 70184, United States; email: bjursic1@uno.edu, tel: 5048580515

\section{Contributions:}

JS: performed and help in design of cell culture analyses, cell toxicity tests, microscopy, imaging, data analyses and preparation of the corresponding figures; CV: assisted JS in cell culture and toxicity experiments; KR: helped designing cell culture experiments, authored manuscript sections related to glioblastoma, cell culture, cell toxicity, and interpretation of the in vitro studies; BSJ: designed chemical strategies for the development of new HR compounds, performed corresponding chemical reactions, computational analyses of the new compounds, authored the section of the manuscript related to development of chemical modifications and computational analyses; AL and KB: performed in vitro BBB penetration assay and HPLC analyses. CF and FP: designed and performed experiments and analyzed the data for all metabolic studies of glioblastoma cells treated with HR compounds. $\mathrm{Chl}$ and SCC: participated in performing specific chemical reactions designed by BSJ, and performed IC50 analyses for some of the HR compounds; SC: editing and conceptual effort related to the final design of the manuscript. All Authors reviewed the manuscript.

\section{Competing Interests}

Disclosure of Potential Conflict of Interest: Dr. Branko Jursic is associated with Stepharm LLC, P.O. Box 24220, New Orleans, LA; Dr. Reiss is associated with WayPath Pharma LLC. 217 Sena Dr. Metairie LA 70005. Dr. Krzysztof Reiss and Dr. Branko Jursic have an LSU patent for the HR compounds presented in this manuscript ("Anticancer Composition and methods of use" 2932719-056-us2). Other authors do not have any competing interest in relation to this submission.

\section{SUPPLEMENTARY INFORMATION}

NMR and MS spectra for all HR compounds generated in this study are included in Supplementary Materials. 


\section{Supplementary Files}

This is a list of supplementary files associated with this preprint. Click to download.

- SupplementaryMaterials091521.pdf 